\title{
Ultra-filtration of human serum for improved quantitative analysis of low molecular weight biomarkers using ATR-IR spectroscopy
}

Franck Bonnier ${ }^{*}, 1$, Hélène Blasco ${ }^{2,3}$, Clément Wasselet $^{1}$, Guillaume Brachet $^{4}$, Renaud Respaud $^{5}$, Luis Felipe CS. Carvalho ${ }^{6}$, Dominique Bertrand ${ }^{7}$, Matthew J. Baker ${ }^{8}$, Hugh J. Byrne ${ }^{9}$, Igor Chourpa $^{1}$

${ }^{1}$ Université François-Rabelais de Tours, Faculté de Pharmacie, EA 6295 Nanomédicaments et Nanosondes, 31 avenue Monge, 37200 Tours, France.

${ }^{2}$ CHRU de Tours, Laboratoire de Biochimie et de Biologie Moléculaire, Tours, France.

${ }^{3}$ INSERM, UMR U930 "Imagerie et Cerveau", Université François Rabelais, Tours, France.

${ }^{4}$ Université François Rabelais de Tours, UMR CNRS 7292 Génétique, Immunothérapie, Chimie et Cancer, Faculté de Médecine, 10 Bd Tonnellé, 37032 Tours Cedex

${ }^{5}$ Université François-Rabelais de Tours, UMR 1100, CHRU de Tours, Service de Pharmacie, F-37032 Tours, France

${ }^{6}$ Universidade do Vale do Paraiba, Laboratory of Biomedical Vibrational Spectroscopy, Sao José dos Campos, Brazil

${ }^{7}$ Data Frame, Nantes, France

${ }^{8}$ WestCHEM, Technology and Innovation Centre, Department of Pure and Applied Chemistry, University of Strathclyde, 295 Cathedral Street, Glasgow G1 1XL, UK

${ }^{9}$ FOCAS Research Institute, Dublin Institute of Technology (DIT), Camden Row, Dublin 8, Ireland

Received zzz, revised zzz, accepted zzz

Published online zzz

Key words: Human serum fractionation, centrifugal filtration, IR spectroscopy, Attenuated Total Reflection (ATR), Partial Least Squares Regression, Low Molecular Weight Fraction (LMWF), Disease diagnosis 


\begin{abstract}
Infrared spectroscopy is a reliable, rapid and cost effective characterisation technique, delivering a molecular finger print of the sample. It is expected that its sensitivity would enable detection of small chemical variations in biological samples associated with disease. ATR-IR is particularly suitable for liquid sample analysis and, although air drying is commonly performed before data collection, just a drop of human serum is enough for screening and early diagnosis. However, the dynamic range of constituent biochemical concentrations in the serum composition remains a limiting factor to the reliability of the technique. Using glucose as a model spike in human serum, it has been demonstrated in the present study that fractionating the serum prior to spectroscopic analysis can considerably improve the precision and accuracy of quantitative models based on the Partial Least Squares Regression algorithm. By depleting the abundant high molecular weight proteins, which otherwise dominate the spectral signatures collected, the ability to monitor changes in the concentrations of the low molecular weight constituents is enhanced. The Root Mean Square Error for the Validation set (RMSEV) has been improved by a factor of 5 following human serum processing with an average relative error in the predictive values below $1 \%$ is achieved. Moreover, the approach is easily transferable to different bodily fluids, which would support the development of more efficient and suitable clinical protocols for exploration of vibrational spectroscopy based ex-vivo diagnostic tools.
\end{abstract}




\section{Introduction}

Bodily fluids have become a much investigated source of samples for the development of rapid and cost effective diagnostic methods ${ }^{1-4}$. Reducing the invasiveness of the current approaches to get molecular information linked to pathological events occurring in different organs affected by various diseases is naturally one of main challenges to improve the patients' comfort. Therefore, performing analysis directly on a few microliters of serum, plasma, saliva or urine has attracted great interest in the medical field, especially due to the facile specimen collection. Blood analysis still holds much promise for accurate ex vivo diagnostics and, compared to deep tissue biopsies, often requiring extreme interventions, the reduced invasiveness of the syringe remains a quite acceptable alternative.

Human serum (or plasma) is a vast reservoir for biochemical products collected and accumulated while perfusing the different organs of the body ${ }^{5}$, ultimately reflecting the physiological status of a patient. It is expected that modifications in its overall composition could indicate the presence of disease and consequently deliver a diagnostic based on specific molecular signatures ${ }^{6}$. It is well accepted that proteins secreted and shed from cells and tissues, such as prostate-specific antigen (PSA) and CA125, can be identified routinely and act as biomarkers of disease ${ }^{7}$. In addition, proteolysis within the tissue or deregulated posttranslational events participate in fragmentation of proteins produced in tumours that diffuse into the circulation ${ }^{6}$. This is further supported by recent associations of pathological conditions with small protein and peptide profiles in serum, notably for diabetes ${ }^{8}$, cardiovascular or infectious diseases ${ }^{9}$. The number of studies related to serum proteomics, peptidomics or metabololics has literally exploded in the literature, complex analytical techniques such as chromatography and/or mass spectroscopy being the reference tools ${ }^{10,11}$. Quite naturally, vibrational spectroscopy, which has been widely employed in biomedical analysis, from tissue sections $^{12,13}$ to single cells ${ }^{14,15}$ with a well demonstrated potential for diagnosis ${ }^{16,17}$, has the capability to become the next generation gold standard tool for serum based patient screening ${ }^{18-}$ ${ }^{20}$. The advent of imaging technologies coupled to the rapid data collection offered by FTIR systems has contributed considerably to the attraction of infrared absorption spectroscopy for biomedical applications ${ }^{21-23}$. However, other methods such as ATR-FTIR are highly suitable for analysis of liquid samples, such as body fluids, allowing delivery of chemical fingerprints from micro-deposition of the samples directly on the Attenuated Total Reflection (ATR) crystal $^{24,25}$. The IR spectrum contains rich and specific information about the molecular composition of the human serum which, coupled to advanced multivariate analysis tools, could deliver accurate diagnostics. Recent systematic studies have demonstrated the potential of IR serum based diagnosis with different data mining approaches such as Principal Components Analysis (PCA), PCA coupled to Linear Discriminant Analysis (PCA-LDA), Random Forest or Support Vector Machine $(\mathrm{SVM})^{26-30}$. However, the development of spectroscopic technologies with medical perspectives needs to align with clinical requirements to enable clinical translation of these promising technologies ${ }^{31}$. Although infrared and Raman spectroscopy have great potential for serum based detection of disease biomarkers ${ }^{32,33}$, in many cases, detection of the presence of a biomarker is not sufficient, but rather quantification within a physiologically relevant range is required, a common example being glucose levels in blood. ${ }^{34}$.

Partial Least Squares Regression analysis (PLSR) remains one of most used analysis methods for quantitative models ${ }^{35}$ either in different body fluids such as urine ${ }^{36}$, saliva ${ }^{37,38}$, serum ${ }^{39}$ or to evaluate physiological responses to drugs in cells ${ }^{40}$. However, the serum composition is highly complex, with up to 10,000 different proteins in an overall concentration ranging from 
60 to $80 \mathrm{mg} / \mathrm{mL}$. Moreover, other circulating molecular species such as sugars, lipids, peptides, metabolites are present, adding to the complexity of the mixture and consequently making quantitative analysis of variations in individual constituents a challenging task. Human serum is also characterized by the dynamic range of concentrations observed between the abundant, high molecular weight (HMW) and the sparse, low molecular weight (LMW) molecules. For example, human serum albumin (HSA) (57-71\%) and globulins (8-26\%) are two abundant HMW proteins completely dominating the composition of the serum quantitatively, potentially impacting the detection and monitoring small variations in the features related to the presence of the informative low molecular weight proteins/peptides/metabolites ${ }^{41}$. The analytical capabilities of traditional proteomic methods are limited, given this large dynamic range of concentrations in the serum. Therefore, depletion of the highly abundant proteins is now recognized as the first step, yet to be optimized, in the analysis of the serum composition ${ }^{42}$. ATR-FTIR suffers from similar limitations to those encountered with chromatography and/or mass spectroscopy based approaches, which can be overcome by means of sample fractionation prior to data recording ${ }^{24}$. Including a separation step prior to IR analysis in order to enhance the specificity and sensitivity have been proposed through coupling with chromatography technologies such as LC-IR and GC-IR ${ }^{43}$. Those hyphenated approaches have been reported in chemistry, pharmaceutical and food sciences applications ${ }^{44-46}$. However, the development of cryogenic temperature control methods during the sample preparation remains the most promising aspect for possible emergence of such technologies in biomedical applications and human body fluids analysis ${ }^{47}$. This present study however investigates the benefits of an alternative approach combining centrifugal filtration/fractionation with ATR-IR spectroscopy for the analysis of human serum as a proof of principle for monitoring and quantified potential low molecular weight biomarkers. Glucose has been used as a model to illustrate the strategy to isolate the relevant fraction of the sample to minimize the influence of the HMW proteins and improve the precision and accuracy of the quantitative models built using the PLSR algorithm. Initially, human serum spiked with systematically varying, physiologically relevant, concentrations of glucose will be used to optimise the measurement protocol, including a study of how the volume deposited on the ATR crystal influences the relevancy of the data collected. PLSR will be employed to build a predictive model over the concentration range to demonstrate the principle of the predictive capacity of the technique and the improved precision afforded by serum fractionation. In the second part of the study, the technique will be demonstrated in patient samples of known varying glucose levels, validating the potential for glucose level monitoring. 


\section{Experimental}

\subsection{Materials and Methods}

Sterile, filtered human serum from normal mixed pool (off the clot) was purchased from TCS Biosciences (UK) for the in vitro model prepared from spiked solutions, while patient serum samples were donated by the University Hospital CHU Bretonneau de Tours (France), following the institutional ethical procedures. Initially, the samples were collected during routine blood check-ups, $1 \mathrm{~mL}$ of the vial remains being provided for further spectroscopic analysis. Commercially available, centrifugal filtering devices, Amicon Ultra-0.5ml (Millipore - Merck, Germany), with cut-off points at $10 \mathrm{kDa}$, were employed to fractionate the serum samples, in both cases. As a result, for each sample, 2 fractions were obtained following filtration; the first representing serum constituents with a molecular weight higher than the cutoff point of the filter used (concentrate); the second corresponding to the fraction passed by the membrane and collected in the vial (filtrate). $0.5 \mathrm{~mL}$ of the serum was placed in the centrifugal filter for spinning. The procedure for washing the centrifugal devices prior to serum processing was adapted from ${ }^{48}$, based on manufacturer's guidelines, and performed as follows: The Amicon Ultra- $0.5 \mathrm{ml}$ filter was spun thrice with a solution of $\mathrm{NaOH}(0.1 \mathrm{M})$, followed by 3 rinses with Milli-Q water (Millipore Elix S). For both washing and rinsing, $0.5 \mathrm{~mL}$ of the respective liquid was added to the filters and the centrifugation was applied for 10 mins at 14 $000 \mathrm{~g}$ followed by a spinning with the devices upside down at $1000 \mathrm{~g}$ for 2 mins in order to remove any residual solution contained in the filter. The aim of the study was to illustrate the potential of ATR-IR spectroscopy to detect and screen a potential biomarker in the LMWF. Therefore, based on previous experience demonstrating that $100 \%$ of the LMWF is recovered with the $10 \mathrm{kDa}$, only this cut-off has been included in the present work $^{24}$.

Additionally, D-Glucose (Fisher scientific, UK) was analysed as reference chemical compound for the quantitative analysis on whole and filtered human serum. Notably, glucose has been selected because it is routinely screened in clinics allowing determination of exact blood levels for each patient sample tested.

\subsection{Glucose Spiked Human Serum model}

The commercial whole human serum was supplemented with known concentrations of glucose; $0.0 \mathrm{mg} . \mathrm{dL}^{-1}$ (control), $20 \mathrm{mg} . \mathrm{dL}^{-1}, 60 \mathrm{mg} . \mathrm{dL}^{-1}, 100 \mathrm{mg} . \mathrm{dL}^{-1}, 140 \mathrm{mg} . \mathrm{dL}^{-1}, 180 \mathrm{mg} . \mathrm{dL}^{-1}, 220$ mg.dL ${ }^{-1}$, in order to explore the dynamic range and sensitivities of the ATR-IR measurement of unprocessed and filtered samples. The concentrations have been selected to cover a wide

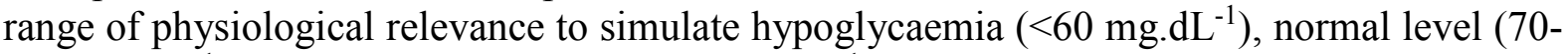
$\left.110 \mathrm{mg} . \mathrm{dL}^{-1}\right)$ and hyperglycaemia $\left(>120 \mathrm{mg} . \mathrm{dL}^{-1}\right)$, in order to optimise the protocols for clinically relevant human serum monitoring using ATR-IR. The serum stock solution has been used as a reference to build the regression models and evaluate the sensitivity of the techniques.

\subsection{Patient samples blood glucose levels}

A total of 15 patient samples have been included in the present study. Glucose levels have been measured at the CHU de Tours using a Cobas analyser following the in house guidelines for routine biochemical analysis. The principle of the test is based on the enzymatic reference method with hexokinase, which catalyses the phosphorylation of glucose to glucose-6phosphate by $\mathrm{ATP}^{49,50}$. Subsequently glucose-6-phosphate is oxidized by glucose-6-phosphate dehydrogenase, in the presence of NADP, to gluconate-6-phosphate. This reaction is specific, 
with no other carbohydrate being oxidized. The rate of NADPH formation during the reaction is directly proportional to the glucose concentration and is measured photometrically in the UV.

Glucose level is one of the most common tests performed during blood check-ups and is therefore a perfect model to develop a proof of principle for quantification of low molecular weight biomarkers in human serum using ATR-IR spectroscopy. The instrumentation available at CHU de Tours has a standard deviation of $0.04 \mathrm{mmol} . \mathrm{L}^{-1}\left(0.721 \mathrm{mg} . \mathrm{dL}^{-1}\right)$, and can therefore be considered as the gold standard. Measured glucose concentrations were provided with the patient samples and have been used as target values for the PLSR models. A summary of information extracted from patient histories is given in Table 1 (see below).

All patient samples have been processed with the $10 \mathrm{kDa}$ centrifugal devices in order to carry out HMWF proteins depletion prior to IR analysis. The reproducibility being a critical point to ensure reliability of the results, a set of 10 independent samples ( 5 unprocessed and 5 filtered mixed pool human serum samples) has been tested on the Cobas analyser before and after ultrafiltration. Ultimately, it has been demonstrated that the concentration of glucose was 4.0 $+/-0.1 \mathrm{mmol} . \mathrm{L}^{-1}$ in both unprocessed and filtered samples, demonstrating that glucose can freely pass through the membrane of the filters and be quantified in the filtrates recovered. This observation correlates with previous testing of reproducibility highlighting the repeatability of the process ${ }^{24}$.

Table 1: Summary of patient's information

\begin{tabular}{|c|c|c|c|c|}
\hline \multirow[b]{2}{*}{$\begin{array}{l}\text { Sample } \\
\text { number }\end{array}$} & \multicolumn{2}{|c|}{ Glucose blood levels } & \multirow[b]{2}{*}{ Gender } & \multirow[b]{2}{*}{ Age } \\
\hline & $\underset{(+/-0.04)}{\operatorname{mmol} . L^{-1}}$ & $\underset{(+/-0.721)}{\operatorname{mg} . \mathrm{dL}^{-1}}$ & & \\
\hline 1 & 3.4 & 61.25 & $\mathrm{~F}$ & 33 \\
\hline 2 & 3.5 & 63.05 & $\mathrm{~F}$ & 36 \\
\hline 3 & 3.6 & 64.86 & $\mathrm{~F}$ & 64 \\
\hline 4 & 3.7 & 66.66 & M & 31 \\
\hline 5 & 3.7 & 66.66 & $\mathrm{~F}$ & 30 \\
\hline 6 & 3.7 & 66.66 & $\mathrm{~F}$ & 87 \\
\hline 7 & 3.8 & 68.46 & $\mathrm{~F}$ & 26 \\
\hline 8 & 6.4 & 115.3 & $\mathrm{M}$ & 73 \\
\hline 9 & 6.5 & 117.10 & $\mathrm{M}$ & 65 \\
\hline 10 & 6.7 & 120.70 & $\mathrm{~F}$ & 58 \\
\hline 11 & 7.0 & 126.11 & $\mathrm{M}$ & 75 \\
\hline 12 & 7.3 & 131.51 & M & 53 \\
\hline 13 & 9.5 & 171.15 & $\mathrm{M}$ & 79 \\
\hline 14 & 10.0 & 180.16 & $\mathrm{~F}$ & 63 \\
\hline 15 & 11.6 & 208.98 & $\mathrm{M}$ & 63 \\
\hline
\end{tabular}

\subsection{Data collection using the ATR-IR}

ATR-IR spectra were recorded using a Bruker vector 22 equipped with a single reflection golden gate ATR accessory (Specac, UK). A diamond top plate with a $45^{\circ} \mathrm{C}$ incident angle was preferred for this study. Penetration depth of the evanescent wave into the sample is both wavenumber and sample dependent, but is typically on the order of $1 \mu \mathrm{m}$. Spectral data were 
the result of 32 scans, with a spectral resolution of $4 \mathrm{~cm}^{-1}$ covering the spectral window 4000 $-600 \mathrm{~cm}^{-1}$. A background spectrum was also recorded in air (32 scans) and automatically ratioed with the sample spectrum by the software. A built-in quality control is automatically performed by the operating system (OPUS software) every day upon system start up, ensuring the ATR accessory is delivering adequate data and no malfunction of the instrument is detected. Liquid human serum solutions: The spectroscopic analysis of samples was performed directly after deposition of a drop on the crystal $(0.1 \mu \mathrm{l}, 0.2 \mu \mathrm{l}, 0.5 \mu \mathrm{L}, 1 \mu \mathrm{L}$ and/or $2 \mu \mathrm{l})$, following air drying. The drying time necessary is directly related to the volume deposited and can be affected by external parameters such as room temperature and humidity, but it generally comprised between 3-5 mins. Moreover, the effectiveness of the air drying has been confirmed in real time following the evolution of the main water band in the range $4000-2500 \mathrm{~cm}^{-1}$ directly on screen. The broad water band, with a maximum absorbance between $3270-3340 \mathrm{~cm}^{-1}$, is a reliable indicator of the dehydration of the sample deposited. The intensity of the band gradually decreases as the sample dries, until an equilibrium is reached, at which point no further spectral evolution is observed. When the signal has been found to be stable for at least 60 seconds, the drop is considered dry and the spectra were collected. Although not yet automated and requiring the constant presence of the operator to monitor the screen, this approach has been found particularly efficient and the complete drying can be unambiguously confirmed in the data collected, as illustrated with the raw spectrum in figure 1B.

At least 5 drops have been measured per sample and sets of 5 spectra have been collected for each drop in order to take into account both instrumental and inter-drop variability In order to reduce the inter-drop size and shape variability, the deposition has been made on clean and dry crystal. It has been observed that the top diamond plate is quite hydrophobic, preventing the drop from spreading out after deposition and maintaining the round shape of the liquid sample. All drops have been administered with micropipettes, perpendicular to the surface, which ultimately results in reproducible circular shaped samples centred on the crystal after air drying. Finally, only 20 spectra (4 drops) have be used for each sample, selecting the data sets with similar raw maximum absorbance indicating the solutions have been deposited similarly on the crystal. Ultimately, a total of 140 spectra were included in the model study with spiked human serum, while 300 spectra were analysed for the 15 patient samples. $2 \mu \mathrm{L}$ has been found to be the maximum realistically usable volume, considering the drying time and the large number of samples tested.

\subsection{Data pre-processing and analysis}

The different pre-processing and data analysis steps were performed using Matlab (Mathworks, USA). Partial Least Squares Regression (PLSR) analysis was exploited as an approach to quantify the spectral variability generated by either the addition of known concentrations of glucose to the human serum or estimate blood glucose levels in patient samples.

The analysis of the spectra collected has been restricted to the finger print region, in which the sugar contribution occurs in IR spectra. The spectra collected from spiked human serum have been either min-max normalized (MMN) at the $1637 \mathrm{~cm}^{-1}$ peak corresponding to the amide I band or processed using baseline correction (rubber-band) followed by vector normalization (VN). The IR data collected being highly reproducible with no sign of strong distortion linked to physical effects such as Mie scattering, the baseline correction has been mainly employed to correct the slight offset observed in the spectra between drops. For instance, the rubber-band algorithm employed has been adapted for use on spectral data ${ }^{51}$. In the present case, only 2 nodes have been defined at $1800 \mathrm{~cm}^{-1}$ and $900 \mathrm{~cm}^{-1}$ for the baseline correction and this consistently for all spectra. To better appreciate the minimal correction applied to the data sets, the process is illustrated in Figure S.1. It should be noted that, following filtration, the spectral 
signature is quite different, due to the depletion of the abundant proteins. Consequently, the typical Amide I and II bands are not observed in the filtered serum, but are replaced by a strong peak at $1591 \mathrm{~cm}^{-1}$ most likely assigned to conjugated $\mathrm{C}=\mathrm{C}$. For consistency, this band, which is the most intense observed in the spectra of the filtered samples, and is also remote from the glucose bands, has been selected for the min-max normalisation.

In an attempt to preserve the information related to raw absorbance intensity, a different method has been employed for the data collected from patient samples. Due to the fact that none of the spectral features can be assumed to be consistent between patients, and thus cannot be used as internal standard, the min-max normalisation on the amide I band was found to be insufficient (data not shown). However, in order to minimise the slight drifts observed the spectral background, it has been preferred to normalised the data at $1780 \mathrm{~cm}^{-1}$, located in a region away from any IR bands of the serum. This approach was evaluated in this study to compensate from the offsets observed in the raw data, restoring a common baseline for the spectra without losing the relation between absorbance and the concentrations of different molecular species. The PLSR model has been built from the pre-processed data sets. The algorithm works in a supervised fashion, whereby all different concentrations are known before running the analysis. Ultimately, the output gives an estimation of the model precision (Root Mean Square Error RMSE) and linearity between the experimental and predicted concentrations $\left(\mathrm{R}^{2}\right)$. In order to validate the robustness of the models, a 20 fold - cross validation loop has been included in the routine. For each iteration, $50 \%$ of the data is randomly selected to constitute the calibration set, while the remaining $50 \%$ are used as a validation set for the quantitative predictions. In the present study, particular attention has been accorded to exploit the entire outcome of the PLSR models. For this, for each iteration of the cross validation the RMSE, $\mathrm{R}^{2}$ and predicted concentrations have been extracted and compiled to calculate mean and standard deviations values. It is also important to note that, PLSR being a supervised algorithm, spectral variations not directly related to the target variable of glucose concentration should have little to no impact on the precision estimated. Also the air drying has been carefully monitored for each sample, and any residual water variations in the data would not be expressed in the dimensions selected for the construction of the quantitative model.

Finally, the 2 main notions developed throughout the study are the precision, which is the variability in the measurements realised, and the accuracy corresponding to the how closely the result of an experiment agrees with the "true" or expected result. While the first can be given by mean of the RMSEV from the PLSR models and different calculation of standard deviation, the latest has been especially used for the patient samples by comparing the relative error (\%) between the reference concentrations results provided by the clinicians (gold standard) and the predicted concentrations estimated by PLSR. 


\section{Results and discussion}

\subsection{Quantification of Glucose levels in spiked human serum}

\section{1.a Selecting volumes deposited}

ATR-FTIR appears to be a convenient, rapid and reliable approach to collecting high quality spectra from highly concentrated liquid samples such as human body fluids and for instance serum $^{52}$. A drop directly deposited onto the crystal can be analysed without prior dilution to avoid saturation effects generally encountered with transmission mode measurements ${ }^{53}$. Despite the rich composition of the human serum, the water contribution remains quite intense in the data gleaned from liquid form samples and air drying remains preferable to visualise all the spectral features ${ }^{54}$. Figure 1 presents ATR-IR spectra collected from an air dried aqueous solution of glucose $\left(10 \mathrm{gdL}^{-1}\right)$ (Figure 1A) and human serum (Figure 1B). Following air drying, the human serum spectrum displays numerous well defined peaks within the $4000-650 \mathrm{~cm}^{-1}$ spectral widow, allowing clear identification of specific features of the human serum, for example those highlighted in grey at $3280 \mathrm{~cm}^{-1}$ (H-O-H stretching), $2957 \mathrm{~cm}^{-1}$ (Asymmetric $\mathrm{CH}_{3}$ stretching), $2920 \mathrm{~cm}^{-1}$ (Asymmetric $\mathrm{CH}_{2}$ stretching), $2872 \mathrm{~cm}^{-1}$ (Symmetric $\mathrm{CH}_{3}$ stretching), $1536 \mathrm{~cm}^{-1}$ (Amide II of proteins), $1453 \mathrm{~cm}^{-1}\left(\mathrm{CH}_{2}\right.$ scissoring), $1394 \mathrm{~cm}^{-1}(\mathrm{C}=\mathrm{O}$ stretch of COO-), $1242 \mathrm{~cm}^{-1}$ (Asymmetric $\mathrm{PO}_{2}$ stretch), $1171 \mathrm{~cm}^{-1}$ (Ester $\mathrm{C}-\mathrm{O}$ asymmetric stretch) and $1080 \mathrm{~cm}^{-1}(\mathrm{C}-\mathrm{O} \text { stretch })^{54-56}$. The spectrum from whole human serum is clearly dominated by the abundant proteins contribution such as albumin and globulins ${ }^{24}$ which swamp the contribution of less represented biomolecules. In comparison, the glucose signature exhibits fewer features, the main peaks being located in the spectral range $1280-800 \mathrm{~cm}^{-1}$, resulting from both the $v(\mathrm{C}-\mathrm{O})$ and $v(\mathrm{C}-\mathrm{O}-\mathrm{C})$ vibrational modes $^{57}$, as identified in Figure $1 \mathrm{~A}$. In the present work, the limited spectral window of $1800-900 \mathrm{~cm}^{-1}$ was chosen to avoid the regions of strong water absorption. Although the spectral response has stabilised, contributions of water are still visible as a broad background in the region of $\sim 3300 \mathrm{~cm}^{-1}$.

The volume deposited can have an influence on the data collected, depending on the concentration of the solutions analysed and the coverage of the crystal achieved. The so-called "coffee ring effect" has been documented extensively in literature ${ }^{54,58}$ and describes the uneven distribution of the constituents following air drying. Commonly, a higher concentration is obtained on the edge of the deposited material which can result in some spectral variability. In complex mixtures such as human serum, different chemical constituents are deposited at different rates, as described by Vroman ${ }^{59}$, resulting in spatially inhomogeneous chemical composition in the dried deposit. Figure 2 presents the dependence of the area under the curve observed in raw data (AUC - between baseline and peak maximum), calculated for the band $1180-955 \mathrm{~cm}^{-1}$, depending on deposited volume, for two different concentrations of glucose solution. The $10 \mathrm{~g} . \mathrm{dL}^{-1}$ solution does not exhibit any noticeable decrease in the AUC between $0.5 \mu \mathrm{L}$ and $2 \mu \mathrm{L}$, explained by a full coverage of the crystal coupled to a saturation of the signal $^{24}$. The notion of saturation is defined by the loss of linearity between the absorbance and the concentrations measured. In contrast to transmission IR spectroscopy, which can deliver absorbance up to 3 with a saturation manifesting itself as highly noisy spectra coupled to plateau effects, ATR-IR spectra intensities are limited by the depth of penetration of the evanescent wave. In the present study and considering the instrumentation used, it has been observed that the maximum absorbance that can possibly be recorded from protein rich samples such as human serum would never exceed 0.45. Following air drying, the Beer-Lambert type dependence of the absorbance on initial sample concentration and length of the optical path 
can both be partially lost and the critical parameter is the amount of matter deposited. In other words, the deposited sample thickness, and therefore absorbance, depends on the concentration of the droplet applied, but if the sample thickness exceeds the evanescent wave penetration depth, no further increase in the absorbance can be observed. However, when reducing the volume below $0.5 \mu \mathrm{L}$, the deposit thickness becomes less than the sampling depth, and the AUC consequently decreases. The AUC pattern for the $0.1 \mathrm{~g} \cdot \mathrm{dL}^{-1}$ is rather different, a maximum intensity being found with $0.2 \mu \mathrm{L}$. This is a result of the coffee ring effect, as the glucose is accumulated in the edges of the drop, outside the field of data collection for larger volumes (Figure 2, $0.5 \mu \mathrm{L}-1 \mu \mathrm{L}-2 \mu \mathrm{L}$ ), thus delivering poor signals, while the $0.2 \mu \mathrm{L}$ deposit is completely contained on the crystal and entirely recorded. The decrease observed for the $0.1 \mu \mathrm{L}$ sample confirms that the drops are smaller than the ATR crystal area. These observations clearly demonstrate the difficulty to accurately estimate the maximum and minimum glucose concentrations that can be measured with IR, due to the different behaviours at low and high concentrations. However, as demonstrated in the supplementary material (Figure S.2), based on the amount of glucose deposited, such limits can be estimated. Also, as the highest concentrations measurable are much greater than any relevant range for clinical applications, with values between $1210 \mathrm{mg} \cdot \mathrm{dL}^{-1}$ and $24205 \mathrm{mg} \cdot \mathrm{dL}^{-1}$ for respectively $2 \mu \mathrm{L}$ and $0.1 \mu \mathrm{L}$ drops, the minimum concentrations are indeed of far more importance. While a $0.1 \mu \mathrm{L}$ drop would be associated with a limit of detection around $15 \mathrm{mg} \cdot \mathrm{dL}^{-1}$, increasing the volume deposited would gradually decrease the minimum concentrations measurable to $7.5 \mathrm{mg}$. $\mathrm{dL}^{-1}, 3$ mg.dL ${ }^{-1}, 1.5 \mathrm{mg} . \mathrm{dL}^{-1}$ and $0.75 \mathrm{mg} . \mathrm{dL}^{-1}$ for respectively $0.2 \mu \mathrm{L}, 0.5 \mu \mathrm{L}, 1 \mu \mathrm{L}$ and $2 \mu \mathrm{L}$. Such observation implies that larger volumes should deliver more accurate outcomes from the analysis, however further investigations of human serum have been performed with both $2 \mu \mathrm{L}$ and $0.2 \mu \mathrm{L}$. Although $0.1 \mu \mathrm{L}$ would have been optimum to ensure the volume deposited is smaller than the crystal size, due to the viscosity of the serum, the smallest volume that could be deposited as drops was $0.2 \mu \mathrm{L}$.

\section{1.b Construction of the quantitative model: Partial Least Square Regression (PLSR)}

Before analysing the patient samples, a quantitative model has been built using the PLSR algorithm and applied to the glucose spiked human serum models in order optimise the protocol for measurement, including deposit volume, and to evaluate the impact of centrifugal filtration on the sensitivity and accuracy of the technique.

The first step of the study was to build a model accurately mirroring clinically relevant blood variations of glucose levels using human serum spiked with D-glucose. For this, different amounts of pure glucose have been added to the human serum stock solution in order to achieve variable concentrations in the physiologically relevant range of $20 \mathrm{mg} \cdot \mathrm{dL}^{-1}-220 \mathrm{mg} \cdot \mathrm{dL}^{-1}$. The normal glucose concentration being between $70 \mathrm{mg} \cdot \mathrm{dL}^{-1}$ and $110 \mathrm{mg} \cdot \mathrm{dL}^{-1}$, simulation for hypoglycaemia and hyperglycaemia have been deliberately included in the set of samples prepared and tested. Figure 3A presents mean ATR-IR spectra collected following air drying from the different solutions. The $\left(1637 \mathrm{~cm}^{-1}\right)$ min-max normalized data exhibit similar profiles, only the region attributed to sugar being affected by the increase of glucose concentration. In Figure 3B, it can be observed how the $1140-950 \mathrm{~cm}^{-1}$ spectral region evolves from the serum stock solution (red) to the highest concentration prepared (cyan), the main features at $1105 \mathrm{~cm}^{-}$ ${ }^{1}, 1078 \mathrm{~cm}^{-1}, 1032 \mathrm{~cm}^{-1}$ and $989 \mathrm{~cm}^{-1}$ systematically increasing with glucose concentration. 
Pre-processed spectra have been analyzed using the PLSR algorithm to determine the relationship between spectral variations and glucose concentrations. The method being supervised, the different concentrations are taken into account during the calculations. The first step generates a scatter plot, (not shown). Figure 4 presents the first two weighting vectors A and $\mathrm{B}$, confirming the discrimination of the data is based on glucose features, which, as demonstrated in Figure 2 and 3, mainly occur in the 1190-950 $\mathrm{cm}^{-1}$ window. The similarities between the 2 weighting vectors support the fact that more than 1 dimension is required to fully describe the spectral variability due to glucose concentrations. Plotting the Root Mean Square Error from the validation set (RMSEV) as displayed in Figure 5 is commonly used to guide the operator in choosing the optimal number of dimensions necessary to reach the best model. In the present case, a 20 fold cross validation has been preferred, leading to the creation of 20 independent calibration/validation sets and thus 20 predictive models. In order to simplify the illustration, the error bars in Figure 5 illustrate the standard deviation calculated between each iteration of the cross validation. As expected, a strong decrease in the RMSEV is observed within the 3 first dimensions, which is normal behaviour for well discriminated data, followed by a stabilisation of the values observed without any further improvement of the model precision. Considering the min-max normalised spectra used for illustration in Figure 5, a minimum is found at $2.078+/-0.252 \mathrm{mg} . \mathrm{dL}^{-1}$, corresponding to 8 dimensions. For consistency between data sets, the minimum found has always been selected to build the predictive models. It should be noted that similar plots can be obtained from the RMSE for the Calibration sets (not shown).

Ultimately, after selection of the optimal number of dimensions for the data set analysed, a predictive model can be built from the PLSR scatter plot (Figure 4), to compare the observations corresponding to the known concentrations of glucose in the samples with the estimated concentrations from the spectral data sets. In the example presented in Figure 6, a really good linearity was reached, with a $\mathrm{R}^{2}$ value of 0.9992 . The standard deviation $\left(2.172 \mathrm{e}^{-4}\right)$ is indicative of good repeatability between the 20 iterations of the cross validation. However, the standard deviation of the RMSEV $\left(0.2526 \mathrm{mg}^{-\mathrm{dL}^{-1}}\right)$ is equal to about $10 \%$ of the mean value, but remains acceptable considering the precision of the model is below the $\mathrm{mg}^{\mathrm{dL}} \mathrm{LL}^{-1}$ range. Moreover, the error bars display no overlapping of data, indicating that each concentration can be unambiguously identified. This approach has been replicated for all data sets collected from the human serum samples spiked with glucose, either unprocessed (whole serum) or following centrifugal filtration with a $10 \mathrm{kDa}$ device. The summary of the PLSR results is presented in Tables 2 and 3.

\section{1.c Summary of results from unprocessed whole human serum spiked wih glucose}

As detailed in Figure 2, the deposition of $2 \mu \mathrm{L}$ and $0.2 \mu \mathrm{L}$ drops has been used to illustrate 2 distinct experimental conditions corresponding to a coverage exceeding the ATR crystal, after air drying of the samples, compared to a coverage less than the recording area. The objective is to better understand how the volume of the deposit can influence the relevancy of the data collected, notably in relation to the coffee ring effect. It has been documented that such an effect can result in variations of the distribution of molecular species.

A direct comparison of the predictive results obtained from $2 \mu \mathrm{L}$ and $0.2 \mu \mathrm{L}$ (Table 2) drops of whole human serum unambiguously demonstrates that the precision of the PLSR models is strongly affected by the size of the drops. The best RMSEV $\left(2.078+/-0.252 \mathrm{mg} \cdot \mathrm{dL}^{-1}\right)$ was achieved with the Min-Max Normalised (MMN) ATR-IR spectra collected from the $2 \mu \mathrm{L}$ drops, while this value is up to 6 times higher for the $0.2 \mu \mathrm{L}$ deposits $\left(12.347+/-1.852 \mathrm{mg}\right.$. $\mathrm{dL}^{-}$ 
$\left.{ }^{1}\right)$. It should be noted that neither reducing the spectral range of the glucose windows (1190$950 \mathrm{~cm}^{-1}$ ) (See Table $2 \mathrm{GLU}$ ) nor using vector normalisation (VN) improves the precision of the predictive models. (See Table $2 \mathrm{VN}$ ) This observation is rather encouraging and supports the notion that, although the samples exhibit a heterogeneous distribution of the different molecular species following air drying, the centre of the drop still delivers quantitative information that can be linearly captured by the PLSR models.

Interestingly, the $0.2 \mu \mathrm{L}$ does not improve the PLSR quantitative model, which suggests that attempting to ensure that the entire sample is deposited on the ATR crystal, with a coverage less than the recording area, is not the best strategy when performing ATR analysis. An experimental limitation of ATR-IR spectroscopy is the absence of a bright field imaging system coupled to the spectrometer, making the visualisation of the drop positions quite difficult, especially with volumes less than $1 \mu \mathrm{L}$. Therefore, it is simply impossible to ensure the $0.2 \mu \mathrm{L}$ samples have been positioned identically on the ATR crystal, explaining the high RMSEV values.

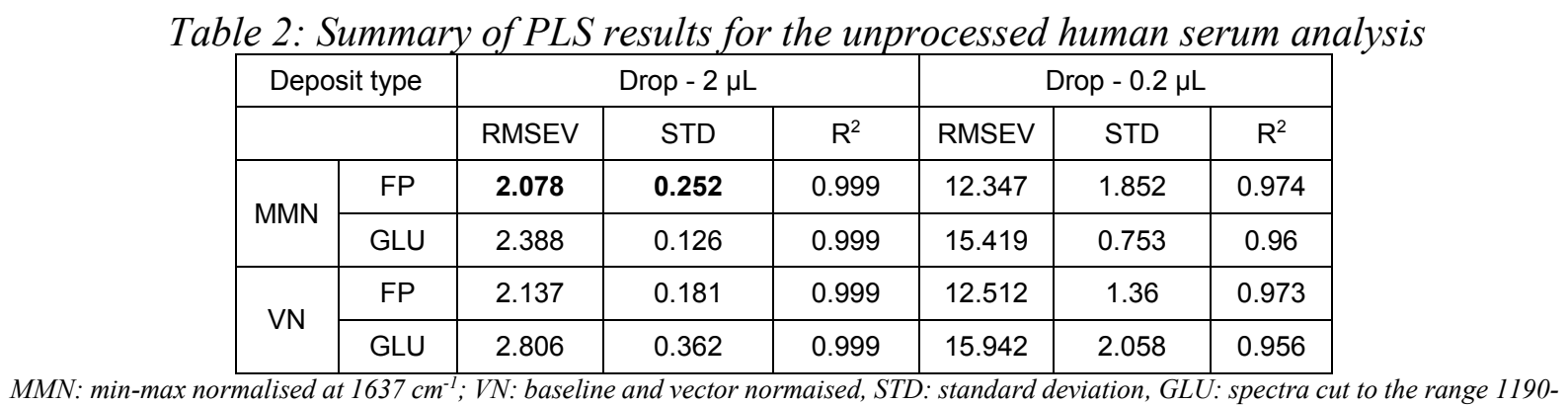

$950 \mathrm{~cm}^{-1}$

\section{1.d Summary of results: 10 kDa filtered human serum spiked with glucose}

Centrifugal filtration has been used to fractionate the human serum and isolate chemical populations depending on their molecular masses. In the present study, a $10 \mathrm{kDa}$ centrifugal device was used in order to specifically deplete the abundant proteins such as globulins and albumin $^{24}$ and retain only the molecular species with low molecular weight in the filtrate, below the cut-off point of the membrane, for subsequent spectroscopic analysis. For instance, glucose is about 180 Daltons, which means it will freely pass through the membrane during spinning of the human serum. Using such an approach, the complex mixture of the human serum can be partially simplified, removing some of the most dominant proteins such as albumin and globulin, consequently enhancing the contribution of underlying constituents in the IR signatures collected as illustrated in Figure 7 . For the $10 \mathrm{kDa}$ filtered serum model, the measurement of deposits from the $2 \mu \mathrm{L}$ drops deliver the best predictive model with a RMSEV value of $2.199 \pm 0.250 \mathrm{mg} . \mathrm{dL}^{-1}$ for the MMN data. As for the unprocessed whole human serum, no improvement can be obtained with deposits from smaller volumes $(0.2 \mu \mathrm{L})$, but rather an increased RMSEV of $5.337 \pm 0.551 \mathrm{mg}^{\mathrm{d} \mathrm{dL}^{-1}}$ is observed, indicating once more that precision in the sample deposition on the ATR crystal is a crucial aspect for this experimental set up. However, as witnessed earlier, the centre of the dried samples preserve the linear relation between glucose concentrations and spectral variations. This is a significant observation as, following the centrifugal filtration, the overall concentration of the serum filtrate is strongly diminished due to depletion of the abundant proteins. The thickness of material deposited from the same volume is therefore significantly reduced, potentially below the region of saturation 
of the STR sampling (Figure 2). The high degree of variability in the raw spectral intensities is compensated by the normalisation of the data prior to PLSR analysis. Ultimately, values of the RMSEV of $2.078 \pm 0.252 \mathrm{mg} . \mathrm{dL}^{-1}$ and $2.199 \pm 0.250 \mathrm{mg} . \mathrm{dL}^{-1}$ for, respectively, the unprocessed and filtered serum are not significantly different, within the standard deviation associated with them (Table 3).

This initial step of the study is rather reassuring, indicating that centrifugal filtration does not affect the sample integrity, which remains representative of the concentrations of the different serum physiological constituents. The main concern was regarding the drying pattern of biological samples characterised by low concentrations, as illustrated in Figure 2, which are more affected by the coffee ring effect which could result in a drastic decrease in the IR spectral intensity due to accumulation of molecules in the edge of the dried drop, with little or no contribution at the centre of it. However, despite the considerably reduced concentration in filtered serum, this effect remains limited and the recordings performed on the $2 \mu \mathrm{L}$ drops are perfectly relevant, delivering good predictive models by means of PLSR analysis. Following those observation, the use of $0.2 \mu \mathrm{L}$ samples can be disregarded for analysis of patient samples, in favour of the $2 \mu \mathrm{L}$. A comparison with a PLSR model constructed from aqueous glucose solutions indicates that the coffee ring effect is limited in filtered serum. In pure glucose solutions, it is observed that the RMSEV tends to be slightly higher than those obtained in Table 3. (See Figure S.4). The small drop in the $\mathrm{R}^{2}$ value (0.9987) illustrates the difficulty to get a linear correlation between spectral variations and concentrations for pure glucose solutions. It also supports the fact that the residual serum constituents present in the $10 \mathrm{kDa}$ filtrates play a key role in maintaining a more consistent drying pattern while reducing the coffee ring effect. Thus, it is important to deplete the human serum and remove the HMW fraction, but preserving the whole LMW fraction seems to be a better approach than trying to further separate or extract glucose from the samples as the surrounding matrix clearly impacts the precision of the measurements done.

Table 3: Summary of PLS results for the 10KDa filtered human serum analysis

\begin{tabular}{|c|c|c|c|c|c|c|c|}
\hline \multicolumn{2}{|c|}{ Deposit type } & \multicolumn{3}{|c|}{ Drop - $2 \mu \mathrm{L}$} & \multicolumn{3}{c|}{ Drop - $0.2 \mu \mathrm{L}$} \\
\hline \multicolumn{2}{|c|}{} & RMSEV & STD & $R^{2}$ & RMSEV & STD & $R^{2}$ \\
\hline \multirow{2}{*}{ MMN } & FP & $\mathbf{2 . 1 9 9}$ & $\mathbf{0 . 2 5 0}$ & 0.999 & 5.337 & 0.551 & 0.995 \\
\cline { 2 - 8 } & GLU & 3.465 & 0.317 & 0.998 & 5.506 & 0.417 & 0.995 \\
\hline \multirow{2}{*}{ VN } & FP & 2.679 & 0.234 & 0.998 & 5.357 & 0.396 & 0.995 \\
\cline { 2 - 8 } & GLU & 4.001 & 0.337 & 0.997 & 8.746 & 0.539 & 0.986 \\
\hline
\end{tabular}

MMN: min-max normalised at $1591 \mathrm{~cm}^{-1} ; V N$ : baseline and vector normaised, STD: standard deviation, GLU: spectra cut in range 1190-950 $\mathrm{cm}^{-1}$

\subsection{Glucose level quantification in patient samples}

Although the quantitative capabilities of ATR-IR spectroscopy can be quite easily demonstrated with model human serum spiked with glucose, both purchased online, the analysis of patient samples can be considered a more delicate matter, the main reason being the multi-parametrical variability observed in clinical applications. When spiking human serum with glucose, only one physiological constituent is affected, while all others remain at similar levels. In that situation, any peak away from the glucose spectral window can be used as an internal standard for calibration of the data, explaining the good results obtained with a MMN to the amide I band. The overall protein content being the same between spiked samples, the 
multivariate analysis of the spectra ultimately highlights the linear evolution of the band ratio between the proteins and glucose. However, samples harvested from patients can display an intrinsic variability, directly reflecting their physiological state on the day. In real conditions, there is no real control, as each individual has its own metabolism, characterised by different ground blood levels for all the serum constituents. Uric Acid, Blood Urea Nitrogen (BUN), Creatinine, total proteins (albumin, globulin), Total Bilirubin, Alkaline Phosphatase, GGTP, LDH, SGOT (also called AST) are some of the most screened serum constituents, although only a few are routinely tested. Glucose is indeed one of them, used for monitoring either hypoglycaemia or hyperglycaemia, particularly important for the detection of diabetes. In addition to the fact that glucose can freely diffuse through the centrifugal device membranes and be fully collected in the filtrate, the choice of the glucose was also motivated because of its physiological relevance and normal levels in human serum. With concentrations ranging between 70-100 mg.dL $\mathrm{dL}^{-1}$, it can be detected in whole unprocessed serum, which was the condition required to be able to build a comparative model between patients samples before and after fractionation.

A total of 15 patient samples have been analysed using ATR-IR spectroscopy, selected to cover a wide range of glucose concentrations mirroring cases of hypoglycaemia and hyperglycaemia (Table 1). The initial measurements have been performed from the unprocessed samples, as provided from the clinician, from $2 \mu \mathrm{L}$ air dried drops. Similarly to the spiked samples, the spectra, min-max normalised at $1780 \mathrm{~cm}^{-1}$, have been analysed with the PLSR algorithm and the results are presented in Figure 9A. With a RMSEV of $11.87+/-0.88 \mathrm{mg} . \mathrm{dL}^{-1}$, the precision of the predictive model has strongly decreased in comparison to the observation made from spiked serum. Furthermore, the $\mathrm{R}^{2}$ reflects the lack of linearity between the spectral variations and the glucose levels, thus the poor quality of the analysis performed. As shown in Figure 8B, although the first (a) and second (b) PLSR weighting vectors contain contributions which can be associated with glucose (Figure 1), there are also significant other contributions associated with serum proteins in the region $1650-1350 \mathrm{~cm}^{-1}$.

Following the first set of measurements, the same patient samples have been processed with a $10 \mathrm{kDa}$ centrifugal device in order to fractionate the serum by depletion of the abundant proteins. From the resulting filtrates, ATR-IR spectra have been recorded, also min-max normalised at the $1780 \mathrm{~cm}^{-1}$ and analysed in identical conditions for comparison purposes. The PLSR model is presented in Figure 9B, displaying a much better RMSEV value $(3.1 \pm 0.13$ mg.dL $\left.{ }^{-1}\right)$. A comparison of both plots unambiguously supports the improvement after centrifugal filtration that can be achieved, confirmed by the reduced standard deviation associated with each concentration tested. Consequently, the linearity of the predictive model is restored to an acceptable value for $\mathrm{R}^{2}$ of 0.9957 . The first (a) PLSR weighting vector, shown in Figure $8 \mathrm{D}$, still contains significant contributions associated with serum proteins in the region $1650-1350 \mathrm{~cm}^{-1}$, although the spectrum (b) of the second is now dominated by glucose. Table 4 gives an overview of the different predicted concentrations for each patient for both unprocessed and fractionised samples. The mean values from the 20 cross validations iterations are given in $\mathrm{mg} / \mathrm{mL}$ with the corresponding standard deviation and finally the relative error compared to the real glucose concentration, as given by the clinician, has been expressed in percentage. Ranging from $16.39 \%$ to $0.25 \%$, the relative error for the unprocessed serum clearly highlights the difficulties to estimate the glucose levels in whole serum. Indeed some of the samples display acceptable mean predictive values because of the supervised fashion of the PLSR algorithm. As for any calibration curve, the trend line illustrates the best fit between data point, meaning few samples are located close to it. In comparison, the filtered serum delivers higher accuracy, ranging from $2.51 \%$ to $0 \%$ mean relative error. Looking at the samples independently, it can be seen that the majority of them are below $1 \%$ relative error, clearly supporting the increased accuracy of the model with fractionated samples. 
Considering the complexity of human serum, concerns can be raised towards the monitoring of low molecular weight physiological constituents potentially relevant for diagnosis. The proof of principle presented throughout this study demonstrates that glucose levels, a wellknown biomarker for diabetes, can be monitored by means of infrared spectroscopy. As highlighted in Figure 1, specific spectral features can be identified for the detection of glucose in complex biological mixtures. Moreover, using multivariate analysis tools such as PLSR, a linear relation between glucose levels and the intensity of glucose spectral features can be obtained. The interferences presented by the HMWF of the human serum is illustrated in the case of patient samples (Figure 9), indicating that the presence of the abundant proteins can be identified as a limiting factor when focusing the spectral analysis of potential low molecular weight biomarkers. Similarly to glucose, the constituents of the LMWF of the serum can exhibit weak contributions in the IR data collected directly impacting on the precision and accuracy of the quantitative models developed. The concept of human serum fractionation proposed in the present work, implies that separating the fractions of interest to perform spectral characterisation independently would ultimately lead to better accuracy. This statement can be further supported by the presence of 3 different patients with similar glucose concentrations as given by the clinicians. Although patients 4, 5 and 6 all have glucose levels found at $66.6 \mathrm{mg} \cdot \mathrm{dL}^{-1}$, all individuals tested would logically present different overall serum composition, notably with consequent variations in the protein contents. While the predictive model built from the whole serum delivers glucose concentrations of respectively $73.44 \mathrm{mg}$. $\mathrm{dL}^{-}$ ${ }^{1}, 66.94 \mathrm{mg} . \mathrm{dL}^{-1}$ and $67.04 \mathrm{mg} \cdot \mathrm{dL}^{-1}$, after depletion of the abundant proteins, those values are found to be $65.66 \mathrm{mg} . \mathrm{dL}^{-1}, 67.16 \mathrm{mg} \cdot \mathrm{dL}^{-1}$ and $67.02 \mathrm{mg} . \mathrm{dL}^{-1}$. Although the analysis is not able to generate exact glucose concentrations for those 3 patients due to the precision of the model, it is noticeable that the inter-individual variability is considerably reduced, leading to improved accuracy. Instrumentation available in clinics present high performance towards glucose level monitoring, and with a standard deviation of $0.72 \mathrm{mg} . \mathrm{dL}^{-1}\left(0.04 \mathrm{mmol} . \mathrm{L}^{-1}\right)$, it is hardly conceivable that IR can deliver better results. Although with a RMSEV of $3.1+/-0.13 \mathrm{mg} . \mathrm{dL}^{-}$ 1 , fractionating the human serum before analysis places the ATR-IR approach in a clinically relevant range of concentrations allowing the identification of patients with abnormal glucose levels (either hypo- or hyper-glycaemia), the aim of the work proposed was to further demonstrate the potential for body fluids screening towards disease diagnosis, in general. However, the precision of the model expressed by the RMSEV indicates the lowest variations in glucose concentration that can be considered statistically relevant for the discrimination of 2 patients with close results. More importantly, the precision and accuracy play key roles in identifying patients at risk, according to blood glucose (BG) falling in the range corresponding to hypoglycaemia or hyperglycaemia. For this reason, the detection and monitoring of biomarkers are subject to requirements specifically defined by the clinical context. For instance, when considering blood glucose monitoring, the concept of the Parkes error grid has been implemented in $1994{ }^{59}$. This model defines performance zones for the results collected, aiming to assess the clinical accuracy of BG monitoring devices. Notably, the Parkes error grid has been introduced as an accepted evaluation tool according to the ISO15197:2013 guideline "In vitro diagnostic test systems -- Requirements for blood-glucose monitoring systems for selftesting in managing diabetes mellitus". Example of Parkes error grids constructed from the PLSR analysis performed from whole and filtered patient serum are given in Figure 10. Each estimated value from both PLSR models (3000 predicted concentrations) has been plotted in order to better visualize their distribution across the risk zones (it should be noted that the model is based on Diabetes type I). While for the whole serum, a majority of the values are found in zone A, defined as the zone of "clinical accurate measurements with no effect on clinical action", it can also be observed that, for the lowest concentrations, many are located in zone B. A general consensus would support that, based on the definition of the risk boundaries, 
a clinically accurate BG meter should show at least $95 \%$ of its data points in zone A of the Parkes error grid. Although the number of patients in the present study remains limited, it is nevertheless interesting to observe the significant improvement obtained after depletion of the abundant proteins by ultrafiltration and how all the predicted concentrations from the PLSR model are now gathered in zone A, unambiguously removing any doubts about the data interpretation (Figure 10B). This further supports the increased clinical relevancy of the quantitative measurement performed after isolation of the LMW fraction. Recently, an increasing numbers of publications promote the capabilities of IR and Raman spectroscopy for serum analysis in both animal and human models from cardiovascular disease to cancers diagnosis $^{61-63}$. However even facing the difficulties to accurately perform specific discrimination or diagnosis ${ }^{2,53}$, resorting to pre-analytical sample preparation procedures is rarely or never considered. Ultimately, the benefits of serum fractionation using centrifugal filtration demonstrated in the case of glucose levels can be easily transferable to any other low molecular weight biomarkers with swapped contribution in the IR data by the HMWF. Indeed, the transferability into clinics remains an open discussion and further in depth investigation is needed to better evaluate the place of centrifugal filtration in medical routines. However, separation techniques such as those based on chromatography hold a pivotal position in diagnosis based on proteomics, peptidomics or metabolomics. Considering the cost to equip a service with an operational LC/MS systems, the labour intensive procedure for sample preparation and analysis and the running costs attached to maintenance of the instrumentation, the IR approach coupled to centrifugal filtration remains a competitive and cost effective alternative, especially in cancer detection. The centrifugal filters can generate enough sample for analysis in less than a minute and, although they can represent an additional cost to patient testing, compared to protocols involving electrophoresis and/or immunoassays, the 3 euros per device (around 3 dollars) could appear to be insignificant. However, this study remains a proof of concept and the emergence of new technologies for automated sample fractionation and protein depletion based on similar membrane based principle may be necessary (e.g. microfluidic based) for translation of such an approach to the clinical environment. Therefore such optimisations would contribute to further broaden the range of applications of vibrational spectroscopic techniques.

Table 4: Summary of PLS results for the unprocessed and $10 \mathrm{kDa}$ filtered human serum patient samples.

\begin{tabular}{|c|c|c|c|c|c|c|c|}
\hline \multirow[b]{3}{*}{ Patient } & \multirow[b]{3}{*}{$\begin{array}{c}\text { Concentrations } \\
(\mathrm{mg} / \mathrm{dL})\end{array}$} & \multicolumn{3}{|c|}{ Unprocessed serum } & \multicolumn{3}{|c|}{$10 \mathrm{kDa}$ Filtered serum } \\
\hline & & \multicolumn{6}{|c|}{ Predicted concentrations (mg/dL) } \\
\hline & & Mean & STD & $\%$ & Mean & STD & $\%$ \\
\hline 1 & 61.25 & 65.39 & 9.84 & 6.75 & 61.68 & 2.45 & 0.71 \\
\hline 2 & 63.05 & 52.71 & 11.46 & 16.39 & 63.05 & 3.74 & 0.00 \\
\hline 3 & 64.86 & 70.34 & 7.59 & 8.45 & 65.37 & 2.73 & 0.78 \\
\hline 4 & 66.66 & 73.44 & 10.69 & 10.17 & 65.66 & 3.04 & 1.51 \\
\hline 5 & 66.66 & 66.94 & 10.98 & 0.42 & 67.16 & 2.68 & 0.76 \\
\hline 6 & 66.66 & 67.04 & 9.90 & 0.58 & 67.02 & 3.66 & 0.55 \\
\hline 7 & 68.46 & 73.71 & 7.62 & 7.67 & 70.18 & 3.33 & 2.51 \\
\hline 8 & 115.3 & 116.54 & 8.49 & 1.08 & 112.92 & 2.65 & 2.06 \\
\hline 9 & 117.1 & 112.04 & 10.04 & 4.32 & 116.30 & 2.42 & 0.69 \\
\hline 10 & 120.7 & 115.32 & 8.68 & 4.46 & 122.65 & 1.90 & 1.61 \\
\hline 11 & 126.11 & 127.20 & 7.44 & 0.86 & 126.64 & 2.89 & 0.42 \\
\hline 12 & 131.51 & 126.72 & 8.91 & 3.64 & 131.43 & 3.39 & 0.06 \\
\hline
\end{tabular}




\begin{tabular}{|l|l|l|l|l|l|l|l|}
\hline 13 & 171.15 & 175.12 & 9.48 & 2.32 & 171.44 & 2.50 & 0.17 \\
\hline 14 & 180.16 & 180.61 & 7.16 & $\mathbf{0 . 2 5}$ & 179.03 & 2.77 & 0.63 \\
\hline 15 & 208.98 & 202.37 & 9.66 & 3.16 & 209.01 & 3.00 & 0.01 \\
\hline
\end{tabular}

STD: standard deviation; \%: relative error between referecence and mean predicted value expressed in percent

\section{Conclusion}

Screening of human serum, and by extension all body fluids, is still an emerging field of application and in order to strengthen the position of Infrared spectroscopy as a potential clinical tool, numerous questions need to be considered and addressed by the community. Firstly, the technique should be competitive with other approaches currently used, such as Mass spectroscopy, in terms of sensitivity and specificity, and, secondly, it should be able to deliver information relevant for clinical diagnosis. Infrared spectroscopy holds immense promise for the implementation of new quantitative analytical techniques in clinical routines. The label free/reagent free argument is often used to further support the relevancy to develop such techniques. However, in order to comply with the high requirements associated with body fluids based diagnostics, a number of improvements of the experimental protocol can be proposed. Notably, delivering quantitative information of the different serum constituents is crucial to validate the approach as a potential clinical tool. Centrifugal filtration of human serum has been proposed to specifically isolate relevant fractions of the samples for more accurate spectroscopic analysis. Using glucose as an example, it has been clearly illustrated that the depletion of the abundant proteins has greatly reduced the spectral variability and consequently significantly improved the precision and accuracy of the quantitative models for potential low molecular weight biomarkers built from the PLSR analysis. In the present study, it has been highlighted that the patients samples are characterised by a normal variability, reflecting the physiological state of the individuals tested, but also that such effects can be considerably reduced by means of easy and rapid pre-analytical samples preparation steps. Furthermore, as the sample processing is also applicable to a wider range of body fluids, the methodology presented in the present work will certainly be beneficial for the field and lead to drastic improvements in the strategies oriented toward their implementation as the next generation of diagnostics techniques clinical tools.

\section{Aknowledgement}

This work was funded in part by the Science Foundation Ireland, Principle Investigator Award 11/PI/1108. 


\section{Figure legends}

Figure 1: Typical ATR IR spectra collected from a $2 \mu \mathrm{L}$ drop of $10 \mathrm{~g} \cdot d L^{-1}$ pure glucose solution deposited on the ATR crystal (A) and human serum (B). Both spectra have been recorded after air drying. No pre-processing has been applied. The spectra have been offset for clarity and the main features of interest in the serum spectrum are highlighted.

Figure 2: Evolution of Area Under the Curve (AUC) of the band at 1180-955 $\mathrm{cm}^{-1}$ as a function of volume of glucose solution deposited. Red: 10 g.dL $L^{-1}$ glucose solution; Blue: 0.1 g.dL $L^{-1}$ glucose solution. Intensity of the blues bars have been multiplied by 5 for better visualization on the graph. Error bars are the results of 5 independent measurements.

Figure 3: Mean ATR-IR spectra collected from unprocessed whole human serum (red) and supplemented with $20 \mathrm{mg} . d L^{-1}$ (blue), $60 \mathrm{mg} \cdot d L^{-1}$ (green), $100 \mathrm{mg} \cdot d L^{-1}$ (yellow), $140 \mathrm{mg} \cdot d L^{-1}$ (black), $180 \mathrm{mg} \cdot d L^{-1}$ (magenta) and $220 \mathrm{mg} . d L^{-1}$ (cyan) of glucose respectively. A: Finger print region 1800-900 $\mathrm{cm}^{-1} ; \boldsymbol{B}:$ Glucose region $1190-900 \mathrm{~cm}^{-1}$. Min-Max normalized spectra on the amide I band $\left(1637 \mathrm{~cm}^{-1}\right)$ used for illustration.

Figure 4: First (A) and second (B) PLS weighting vector corresponding respectively to dimension 1 and 2 of the scatter plot. Min-Max normalized spectra on the amide I band $\left(1637 \mathrm{~cm}^{-1}\right)$ used for illustration.

Figure 5: Evolution of the root mean square error on the validation set (RMSEV) according to the number of dimensions selected in the PLS model. Value are average calculated from the 20 iteration of the cross validation associated to corresponding error bar illustrating the standard deviation. Min-Max normalized spectra on the amide I band $\left(1637 \mathrm{~cm}^{-1}\right)$ used for illustration.

Figure 6: Predictive model build from the PLS analysis. For each concentrations the value displayed is an average of the concentration predicted with the corresponding standard deviation calculated from the 20 iterations of the cross validation. Mean RMSEV and $R^{2}$ values are given on the plot both also with their respective standard deviation. Min-Max normalized spectra on the amide I band $\left(1637 \mathrm{~cm}^{-1}\right)$ used for illustration.

Figure 7: Mean ATR-IR spectra collected from human serum stock solution unprocessed whole (A) and $10 \mathrm{kDa}(B)$ in the finger print region 1800-900 $\mathrm{cm}^{-1}$. Spectra offset for clarity

Figure 8: Evolution of the root mean square error on the validation set (RMSEV) according to the number of dimensions selected in the PLS model with corresponding first (a) and second (b) PLS weighting vector corresponding respectively to dimension 1 and 2 of PLSR model. A: unprocessed serum; B: $10 \mathrm{kDa}$ filtered serum.

Figure 9: A: Predictive model built from the PLS analysis of patient unprocessed samples; B: Predictive model built from the $P L S$ analysis of patient $10 \mathrm{kda}$ filtered samples. For each concentrations the value displayed is an average of the concentration predicted with the corresponding standard deviation calculated from the 20 iterations of the cross validation. Mean RMSEV and $R^{2}$ values are given on the plot both also with their respective standard deviation. Min-Max normalized at $1780 \mathrm{~cm}^{-1}$.

Figure 10: Parkes error grid for patient serum samples. A: Predicted values obtained from the PLSR analysis performed on the whole human serum from patients. B: Predicted values obtained from filtered human serum. Labels A-E define the risks zone restricted to the range $0-250 \mathrm{mg} / \mathrm{dL}$ 


\section{Bibliography}

1. M. Arellano, J. Jiang, X. Zhou, L. Zhang, H. Ye, D. T. Wong and S. Hu, Front Biosci (Schol Ed), 2009, 1, 296-303.

2. M. J. Baker, S. R. Hussain, L. Lovergne, V. Untereiner, C. Hughes, R. A. Lukaszewski, G. Thiefin and G. D. Sockalingum, Chemical Society reviews, 2016, 45, 1803-1818.

3. S. M. Hanash, C. S. Baik and O. Kallioniemi, Nat Rev Clin Oncol, 2011, 8, 142-150.

4. C. E. Thomas, W. Sexton, K. Benson, R. Sutphen and J. Koomen, Cancer Epidemiol Biomarkers Prev, 2010, 19, 953-959.

5. D. W. Greening and R. J. Simpson, Journal of proteomics, 2010, 73, 637-648.

6. A. Tessitore, A. Gaggiano, G. Cicciarelli, D. Verzella, D. Capece, M. Fischietti, F. Zazzeroni and E. Alesse, Int J Proteomics, 2013, 2013, 125858.

7. R. S. Tirumalai, K. C. Chan, D. A. Prieto, H. J. Issaq, T. P. Conrads and T. D. Veenstra, Mol Cell Proteomics, 2003, 2, 1096-1103.

8. D. Basso, A. Valerio, R. Seraglia, S. Mazza, M. G. Piva, E. Greco, P. Fogar, N. Gallo, S. Pedrazzoli, A. Tiengo and M. Plebani, Pancreas, 2002, 24, 8-14.

9. R. B. Rubin and M. Merchant, American clinical laboratory, 2000, 19, 28-29.

10. T. Kimhofer, H. Fye, S. Taylor-Robinson, M. Thursz and E. Holmes, British journal of cancer, 2015, 112, 1141-1156.

11. D. B. Liesenfeld, N. Habermann, R. W. Owen, A. Scalbert and C. M. Ulrich, Cancer Epidemiol Biomarkers Prev, 2013, 22, 2182-2201.

12. S. M. Ali, F. Bonnier, K. Ptasinski, H. Lambkin, K. Flynn, F. M. Lyng and H. J. Byrne, Analyst, 2013, 138, 3946-3956.

13. L. M. Fullwood, D. Griffiths, K. Ashton, T. Dawson, R. W. Lea, C. Davis, F. Bonnier, H. J. Byrne and M. J. Baker, Analyst, 2014, 139, 446-454.

14. J. Dorney, F. Bonnier, A. Garcia, A. Casey, G. Chambers and H. J. Byrne, Analyst, 2012, 137, 1111-1119.

15. Z. Farhane, F. Bonnier, A. Casey, A. Maguire, L. O'Neill and H. J. Byrne, Analyst, 2015, 140, 5908-5919.

16. L. F. Carvalho, F. Bonnier, K. O'Callaghan, J. O'Sullivan, S. Flint, H. J. Byrne and F. M. Lyng, Exp Mol Pathol, 2015, 98, 502-509.

17. F. M. Lyng, D. Traynor, I. R. Ramos, F. Bonnier and H. J. Byrne, Anal Bioanal Chem, 2015, 407, 8279-8289.

18. I. Taleb, G. Thiefin, C. Gobinet, V. Untereiner, B. Bernard-Chabert, A. Heurgue, C. Truntzer, P. Hillon, M. Manfait, P. Ducoroy and G. D. Sockalingum, Analyst, 2013, 138, 4006-4014.

19. J. R. Hands, G. Clemens, R. Stables, K. Ashton, A. Brodbelt, C. Davis, T. P. Dawson, M. D. Jenkinson, R. W. Lea, C. Walker and M. J. Baker, Journal of neuro-oncology, 2016, 127, 463-472.

20. C. Hughes, G. Clemens, B. Bird, T. Dawson, K. M. Ashton, M. D. Jenkinson, A. Brodbelt, M. Weida, E. Fotheringham, M. Barre, J. Rowlette and M. J. Baker, Scientific reports, 2016, 6, 20173.

21. G. Bellisola and C. Sorio, Am J Cancer Res, 2012, 2, 1-21.

22. B. R. Wood, M. Kiupel and D. McNaughton, Vet Pathol, 2014, 51, 224-237.

23. M. Pilling and P. Gardner, Chemical Society reviews, 2016, 45, 1935-1957.

24. F. Bonnier, G. Brachet, R. Duong, T. Sojinrin, R. Respaud, N. Aubrey, M. J. Baker, H. J. Byrne and I. Chourpa, J Biophotonics, 2016, DOI: 10.1002/jbio.201600015. 
25. C. M. Orphanou, L. Walton-Williams, H. Mountain and J. Cassella, Forensic science international, 2015, 252, e10-16.

26. E. Staniszewska-Slezak, A. Fedorowicz, K. Kramkowski, A. Leszczynska, S. Chlopicki, M. Baranska and K. Malek, Analyst, 2015, 140, 2273-2279.

27. G. L. Owens, K. Gajjar, J. Trevisan, S. W. Fogarty, S. E. Taylor, B. Da Gama-Rose, P. L. Martin-Hirsch and F. L. Martin, J Biophotonics, 2014, 7, 200-209.

28. J. Ollesch, S. L. Drees, H. M. Heise, T. Behrens, T. Bruning and K. Gerwert, Analyst, 2013, 138, 4092-4102.

29. D. Perez-Guaita, J. Kuligowski, S. Garrigues, G. Quintas and B. R. Wood, Analyst, 2015, 140, 2422-2427.

30. H. J. Byrne, P. Knief, M. E. Keating and F. Bonnier, Chemical Society reviews, 2015, DOI: $10.1039 / \mathrm{c} 5 \mathrm{cs} 00440 \mathrm{c}$.

31. H. J. Byrne, M. Baranska, G. J. Puppels, N. Stone, B. Wood, K. M. Gough, P. Lasch, P. Heraud, J. Sule-Suso and G. D. Sockalingum, Analyst, 2015, 140, 2066-2073.

32. K. Kong, C. Kendall, N. Stone and I. Notingher, Adv Drug Deliv Rev, 2015, 89, 121134.

33. C. Lacombe, V. Untereiner, C. Gobinet, M. Zater, G. D. Sockalingum and R. Garnotel, Analyst, 2015, 140, 2280-2286.

34. S. Yotsukura and H. Mamitsuka, Critical reviews in oncology/hematology, 2015, 93, 103-115.

35. S. Wold, M. Sjostrom and L. Eriksson, Chemometrics and Intelligent Laboratory Systems, 2001, 109-130.

36. H. M. Heise, G. Voigt, P. Lampen, L. Küpper, S. Rudloff and G. Werner, Applied spectroscopy, 2001, 55, 434-443.

37. A. R. Shaw and H. H. Mantsch, Infrared Spectroscopy in Clinical and Diagnostic Analysis, John Wiley \& Sons Ltd, Chichester., 2006.

38. S. Khaustova, M. Shkurnikov, E. Tonevitsky, V. Artyushenko and A. Tonevitsky, Analyst, 2010, 135, 3183-3192.

39. I. Elsohaby, J. T. McClure, C. B. Riley, R. A. Shaw and G. P. Keefe, Journal of veterinary diagnostic investigation : official publication of the American Association of Veterinary Laboratory Diagnosticians, Inc, 2016, 28, 30-37.

40. H. Nawaz, F. Bonnier, A. D. Meade, F. M. Lyng and H. J. Byrne, Analyst, 2011, 136, 2450-2463.

41. F. Di Girolamo, J. Alessandroni, P. Somma and F. Guadagni, Journal of proteomics, 2010, 73, 667-677.

42. J. L. Luque-Garcia and T. A. Neubert, J Chromatogr A, 2007, 1153, 259-276.

43. K. N. Patel, J. K. Patel, M. P. Patel, G. C. Rajput and H. A. Patel, Pharm Methods, 2010, 1, 2-13.

44. A. Ioannou and C. Varotsis, Journal of Physical Chemistry \& Biophysics, 2016, 6, 210.

45. J. Kuligowski, M. Cascant, S. Garrigues and M. de la Guardia, Talanta, 2012, 99, 660667.

46. A. Edelmann, J. Diewok, J. R. Baena and B. Lendl, Anal Bioanal Chem, 2003, 376, 9297.

47. S. V. Patil, Dr. Shashikant and D. Barhate, World Journal of Pharmaceutical Research, 2014, 4, 214-225.

48. F. Bonnier, M. J. Baker and H. J. Byrne, Anal. Methods, 2014, 6.

49. Kunst A, Draeger B and Z. J., in Methods of Enzymatic Analysis, ed. Bergmeyer, 3rd edn., 1984, vol. VI, pp. 163-172.

50. T. NW, Clinical Guide to Laboratory Tests, Philadelphia. WB Saunders Company,, 4th edn., 2006. 
51. K. Peter, Dublin Institute of technology, 2010.

52. K. M. Dorling and M. J. Baker, Trends Biotechnol, 2013, 31, 327-328.

53. C. Hughes, M. Brown, G. Clemens, A. Henderson, G. Monjardez, N. W. Clarke and P. Gardner, J Biophotonics, 2014, 7, 180-188.

54. F. Bonnier, F. Petitjean, M. J. Baker and H. J. Byrne, J Biophotonics, 2014, 7, 167-179.

55. J. R. Hands, P. Abel, K. Ashton, T. Dawson, C. Davis, R. W. Lea, A. J. McIntosh and M. J. Baker, Anal Bioanal Chem, 2013, 405, 7347-7355.

56. J. R. Hands, K. M. Dorling, P. Abel, K. M. Ashton, A. Brodbelt, C. Davis, T. Dawson, M. D. Jenkinson, R. W. Lea, C. Walker and M. J. Baker, J Biophotonics, 2014, 7, 189199.

57. C. Petibois, A. M. Melin, A. Perromat, G. Cazorla and G. Deleris, The Journal of laboratory and clinical medicine, 2000, 135, 210-215.

58. L. Lovergne, G. Clemens, V. Untereiner, R. A. Lukaszweski, G. D. Sockalingum and M. J. Baker, Anal. Methods, 2015, 7, 7140-7149.

59. L. Vroman, A. L. Adams, G. C. Fischer and P. C. Munoz, Blood, 1980, 55, 156-159.

60. J. L. Parkes, S. L. Slatin, S. Pardo and B. H. Ginsberg, Diabetes Care, 2000, 23, $1143-$ 1148.

61. A. Sahu, S. Sawant, H. Mamgain and C. M. Krishna, Analyst, 2013, 138, 4161-4174.

62. S. L. Haas, R. Muller, A. Fernandes, K. Dzeyk-Boycheva, S. Wurl, J. Hohmann, S. Hemberger, E. Elmas, M. Bruckmann, P. Bugert and J. Backhaus, Applied spectroscopy, 2010, 64, 262-267.

63. Backhausa J, Muellera R, Formanskia N, Szlamaa N, Meerpohlb H.G, Eidtb M and B. P, Vibrational Spectroscopy, 2010, 52, 173-177. 


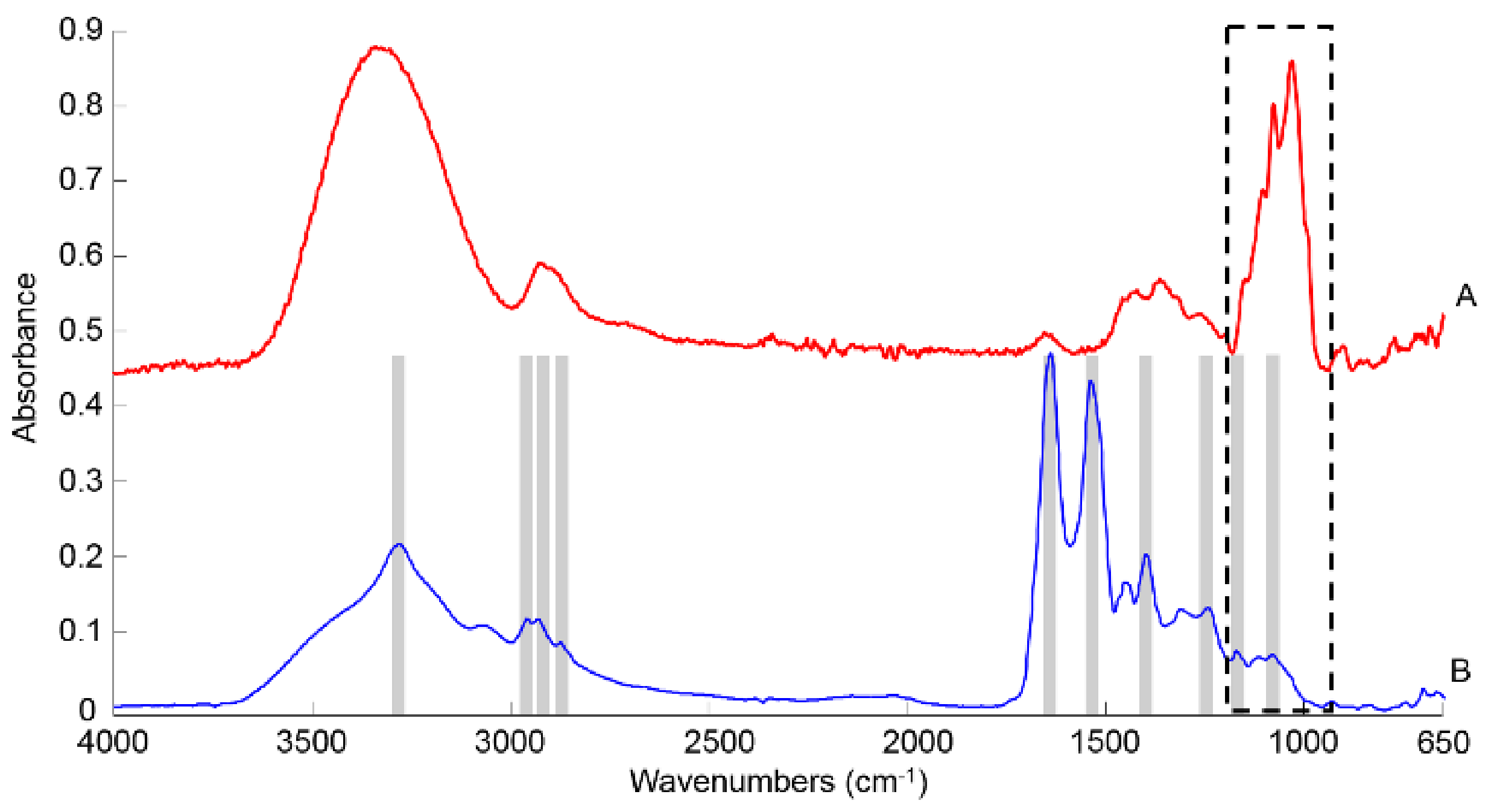

Figure 1: Typical ATR IR spectra collected from a $2 \mu \mathrm{L}$ drop of $10 \mathrm{~g} . \mathrm{dL}-1$ pure glucose solution deposited on the ATR crystal (A) and human serum (B). Both spectra have been recorded after air drying. No pre-processing has been applied. The spectra have been offset for clarity and the main features of interest in the serum spectrum are highlighted. 


\section{0}

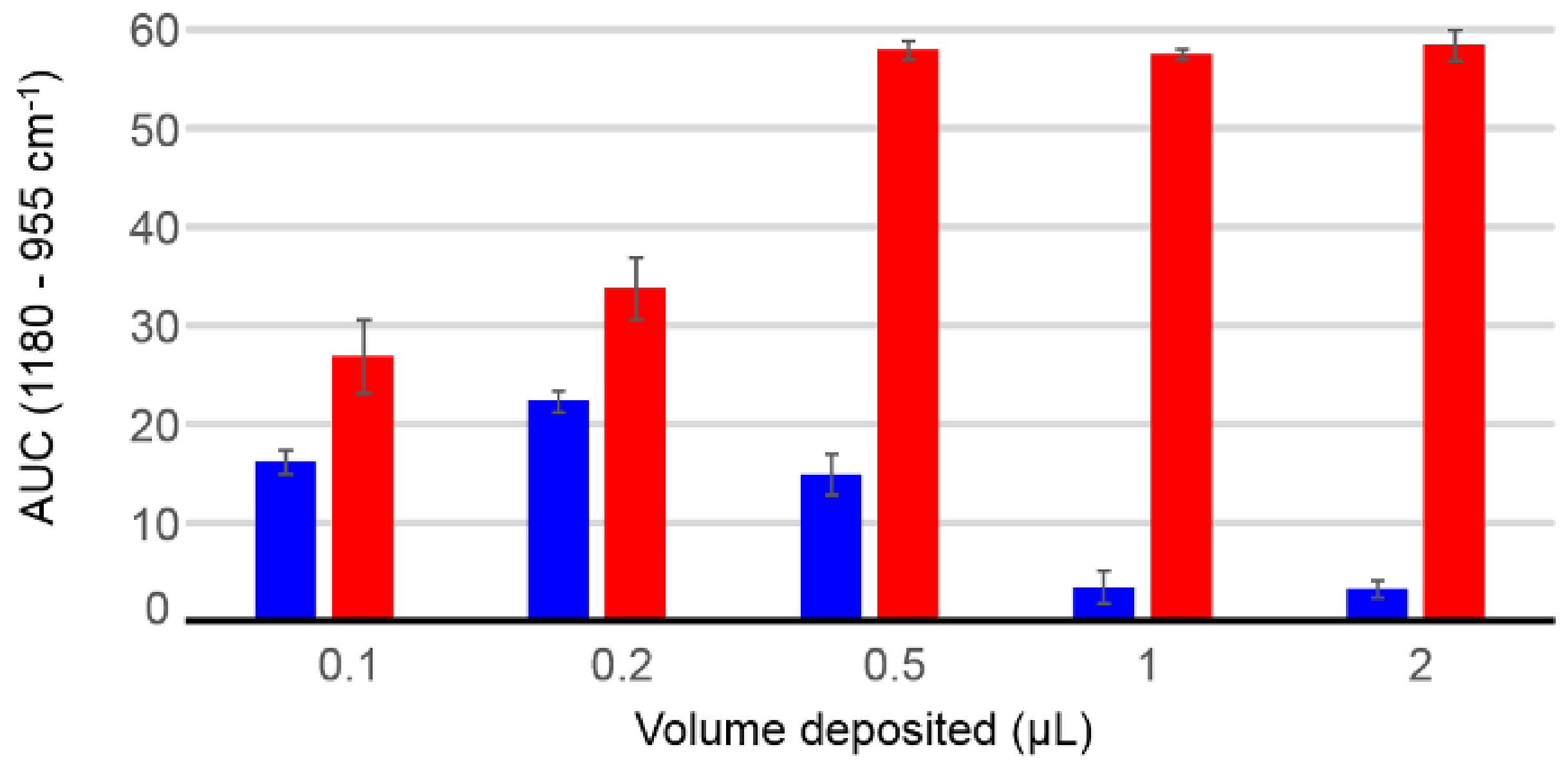

Figure 2: Evolution of Area Under the Curve (AUC) of the band at 1180-955 cm-1 as a function of volume of glucose solution deposited. Red: 10 g.dL-1 glucose solution; Blue: 0.1 g.dL-1 glucose solution. Intensity of the blues bars have been multiplied by 5 for better visualization on the graph. Error bars are the results of 5 independent measurements. 

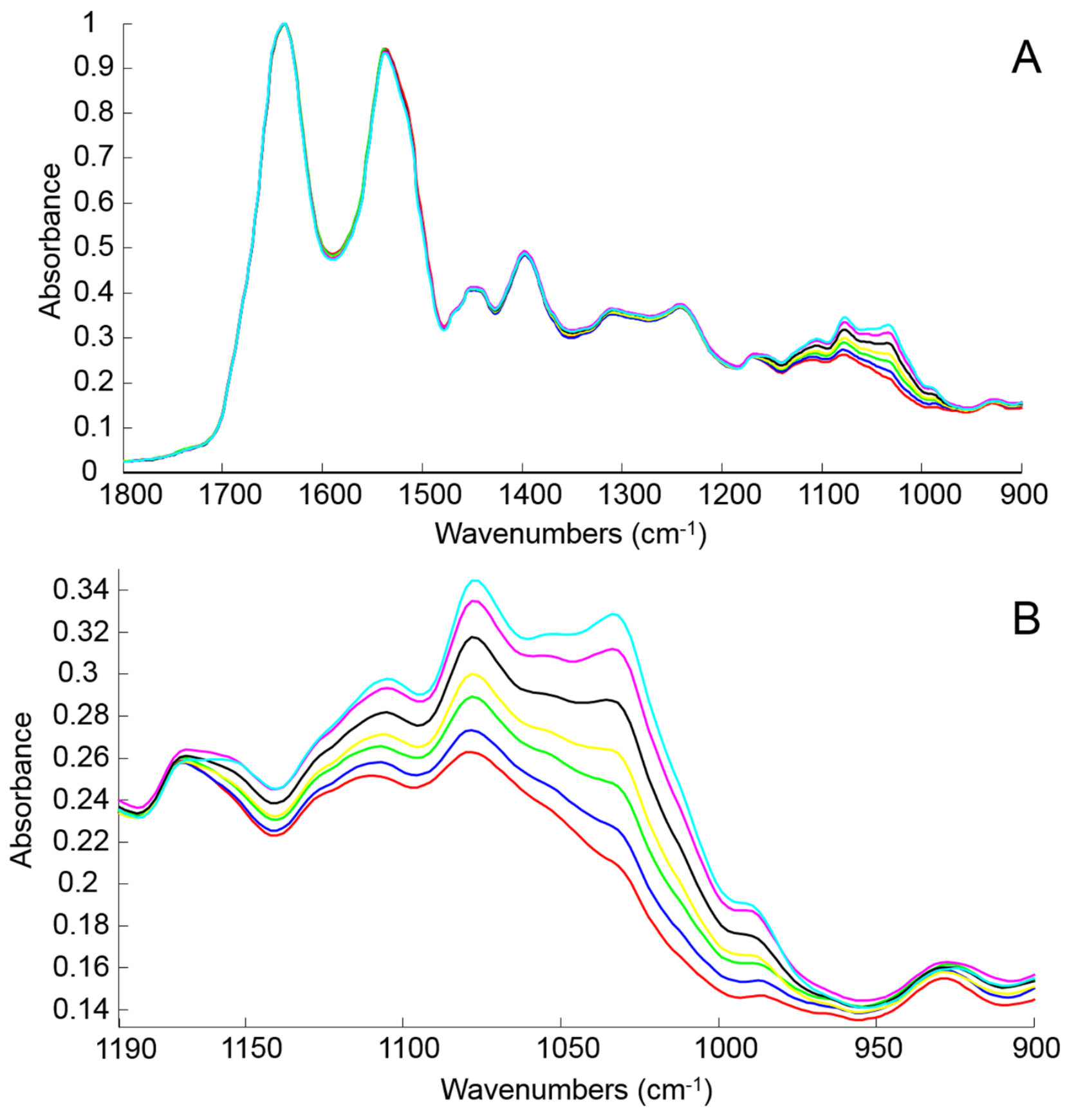

Figure 3: Mean ATR-IR spectra collected from unprocessed whole human serum (red) and supplemented with $20 \mathrm{mg} . \mathrm{dL}-1$ (blue), $60 \mathrm{mg} \cdot \mathrm{dL}-1$ (green), $100 \mathrm{mg} . \mathrm{dL}-1$ (yellow), $140 \mathrm{mg} . d \mathrm{~L}-1$ (black), $180 \mathrm{mg} . \mathrm{dL}-1$ (magenta) and $220 \mathrm{mg} . \mathrm{dL}-1$ (cyan) of glucose respectively. A: Finger print region 1800-900 cm-1; B: Glucose region $1190-900 \mathrm{~cm}-1$. Min-Max normalized spectra on the amide I band (1637 cm-1) used for illustration. 


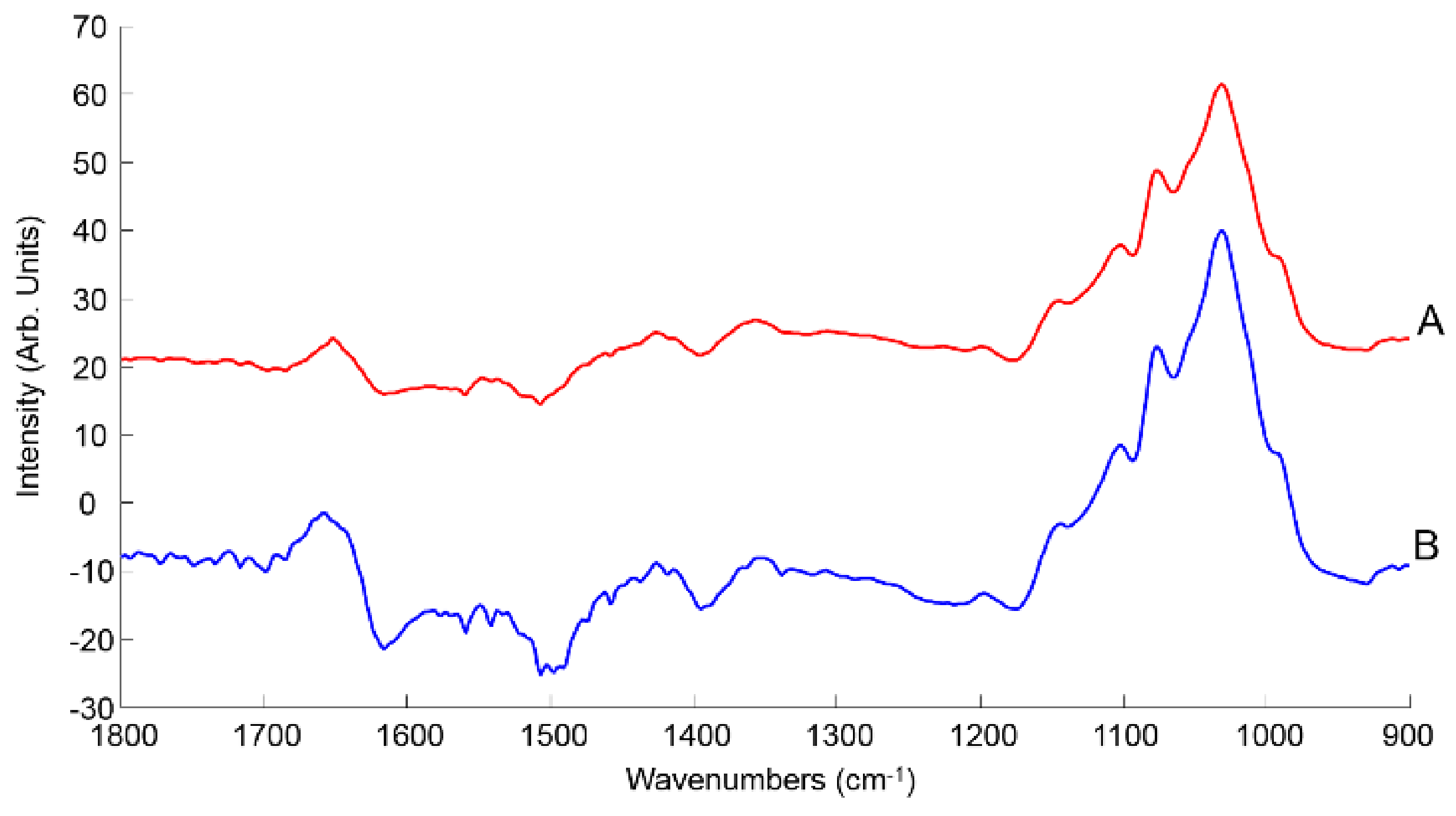

Figure 4: First (A) and second (B) PLS weighting vector corresponding respectively to dimension 1 and 2 of the scatter plot. Min-Max normalized spectra on the amide I band (1637 cm-1) used for illustration. 


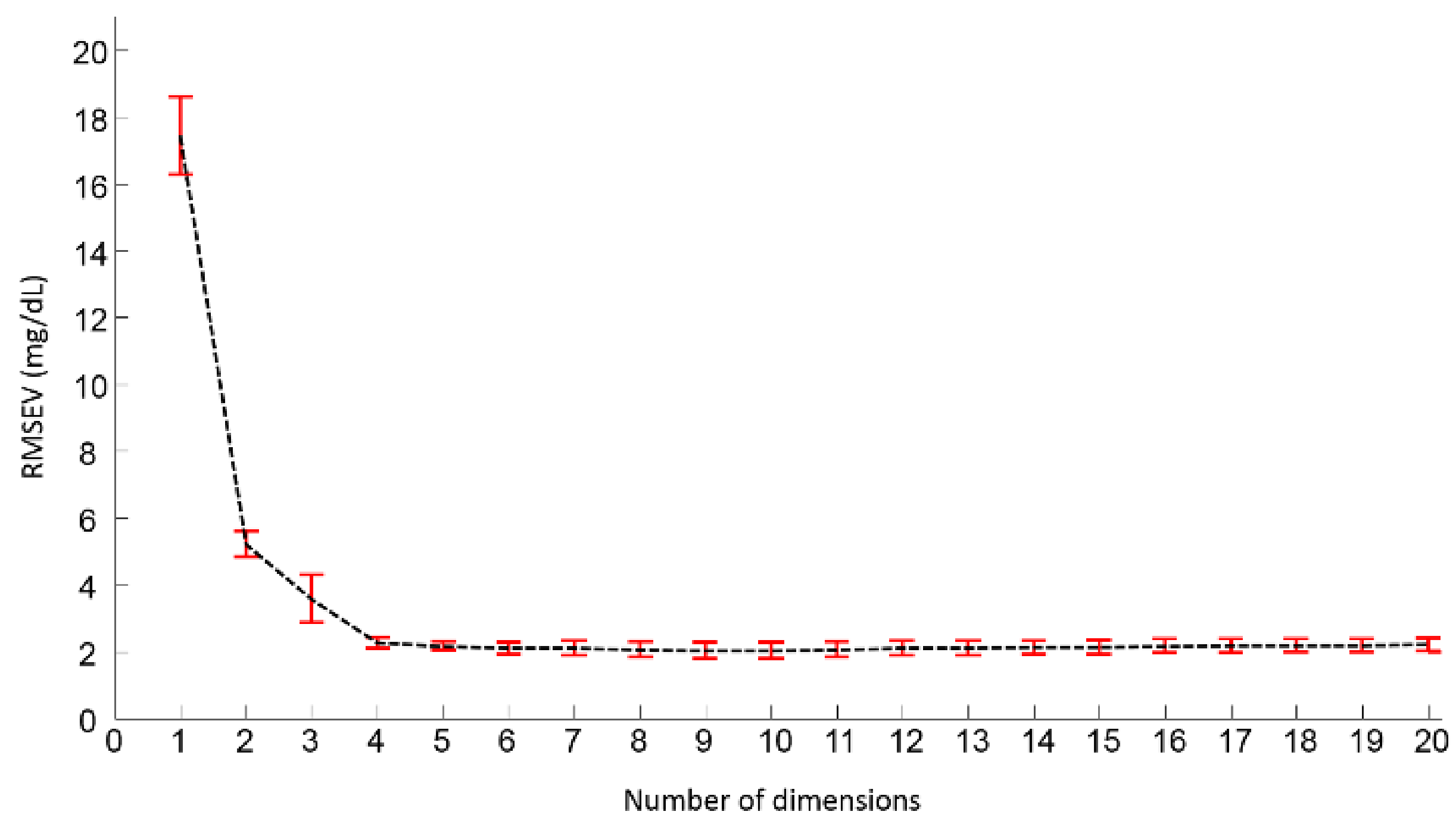

Figure 5: Evolution of the root mean square error on the validation set (RMSEV) according to the number of dimensions selected in the PLS model. Value are average calculated from the 20 iteration of the cross validation associated to corresponding error bar illustrating the standard deviation. Min-Max normalized spectra on the amide I band (1637 cm-1) used for illustration. 


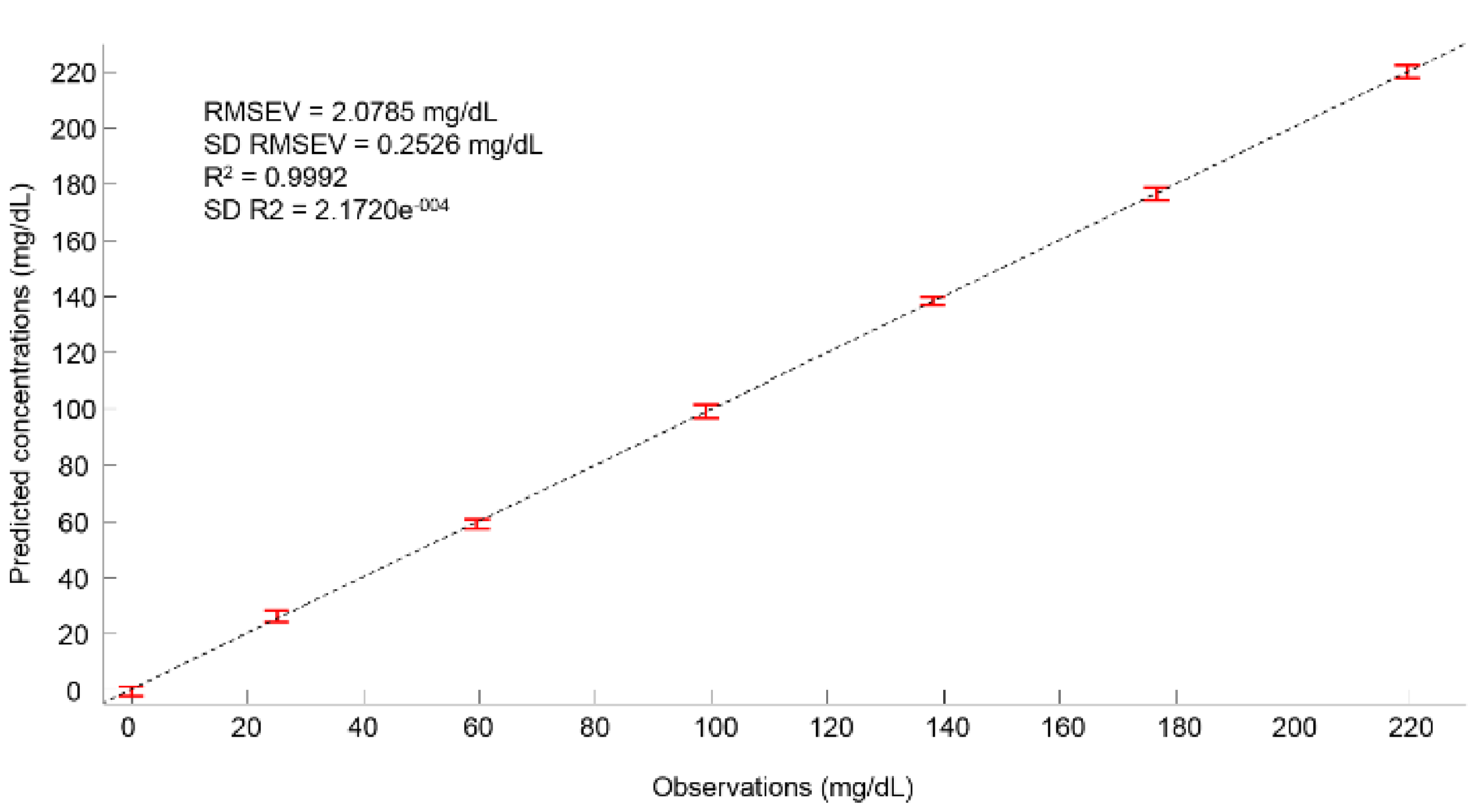

Figure 6: Predictive model build from the PLS analysis. For each concentrations the value displayed is an average of the concentration predicted with the corresponding standard deviation calculated from the 20 iterations of the cross validation. Mean RMSEV and R2 values are given on the plot both also with their respective standard deviation. Min-Max normalized spectra on the amide I band (1637 cm-1) used for illustration. 


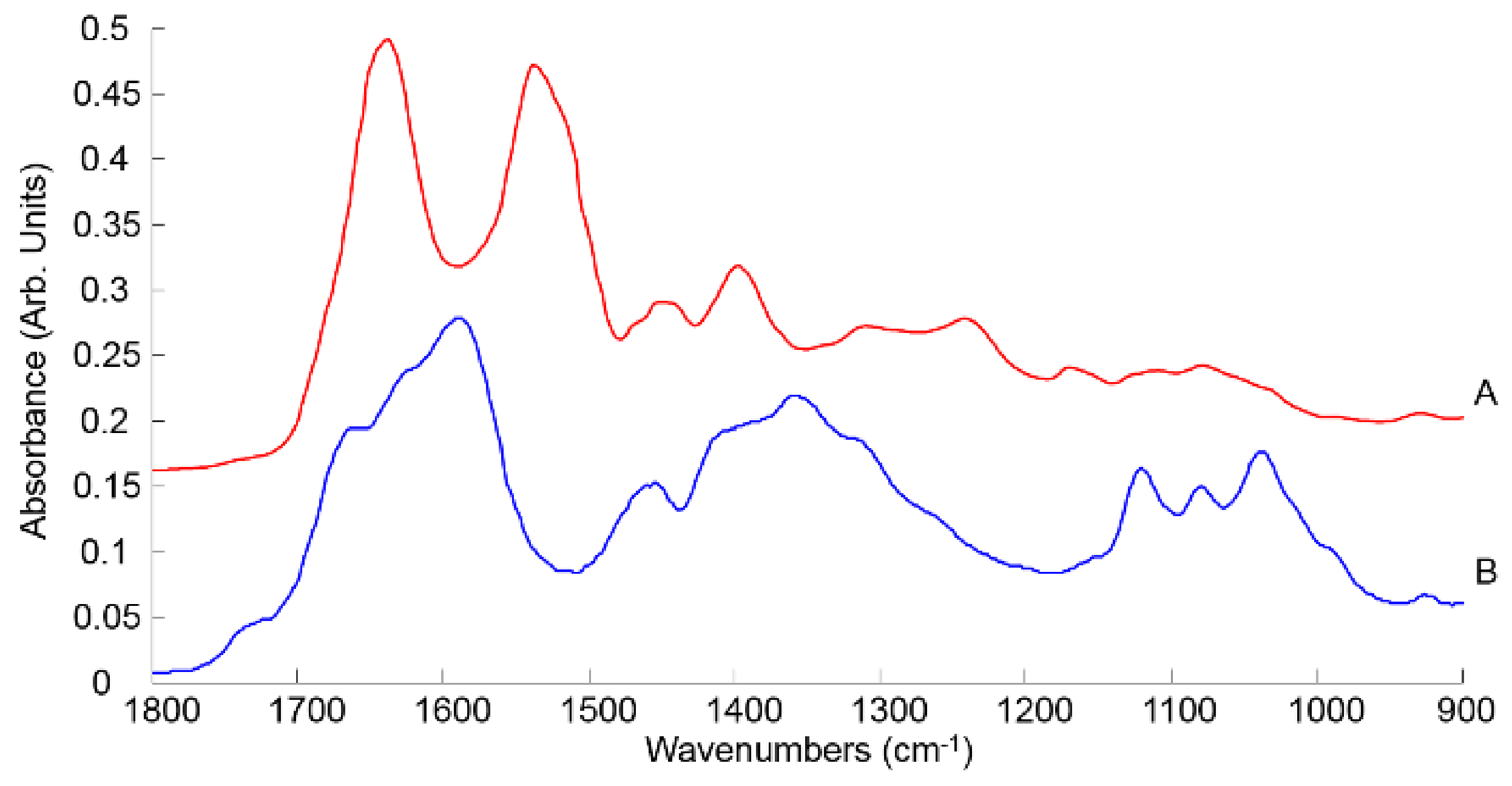

Figure 7: Mean ATR-IR spectra collected from human serum stock solution unprocessed whole (A) and $10 \mathrm{kDa}(B)$ in the finger print region $1800-900 \mathrm{~cm}-1$. Spectra offset for clarity 

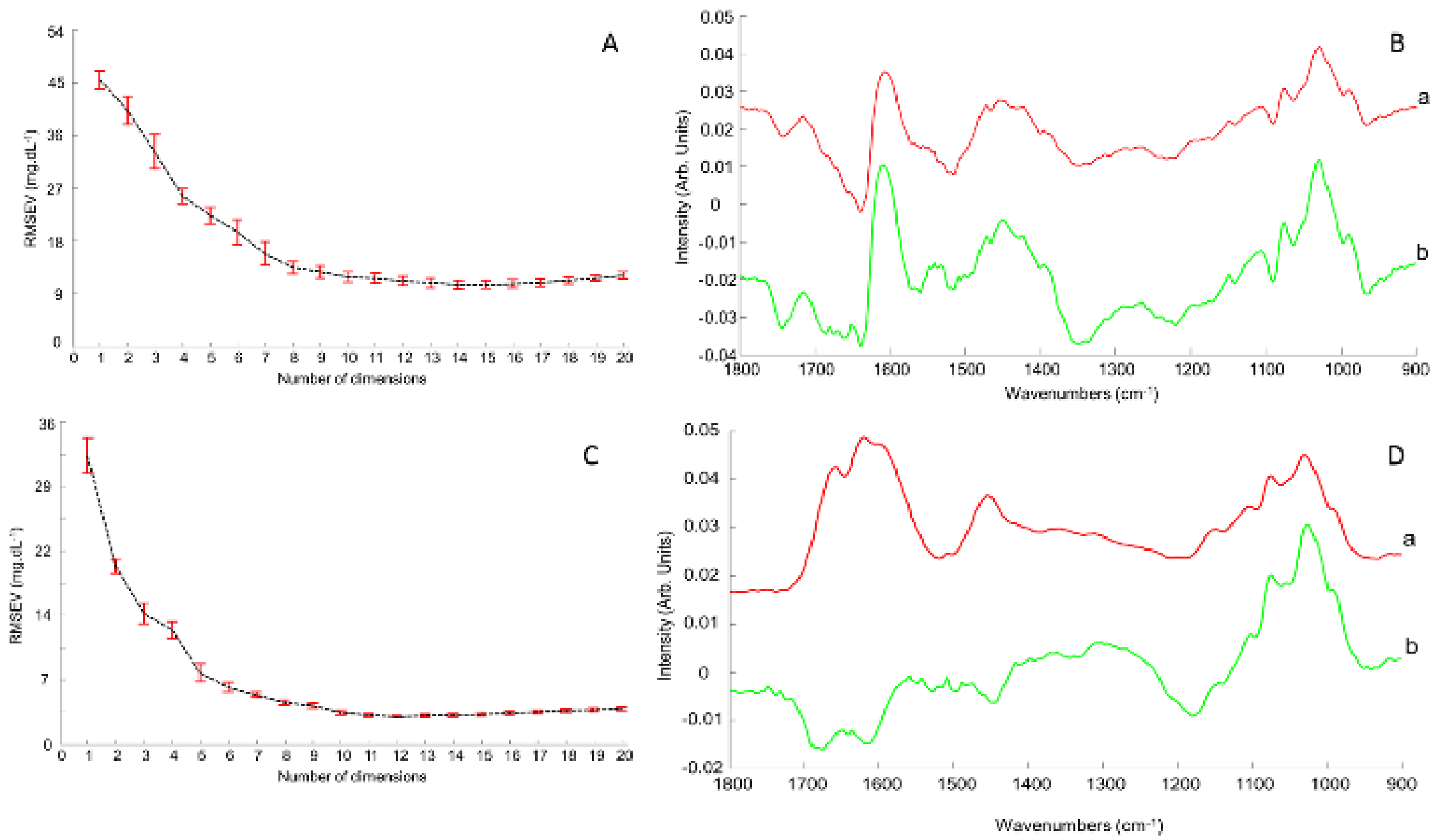

Figure 8: Evolution of the root mean square error on the validation set (RMSEV) according to the number of dimensions selected in the PLS model with corresponding first (a) and second (b) PLS weighting vector corresponding respectively to dimension 1 and 2 of PLSR model. A: unprocessed serum; B: 10 kDa filtered serum. 

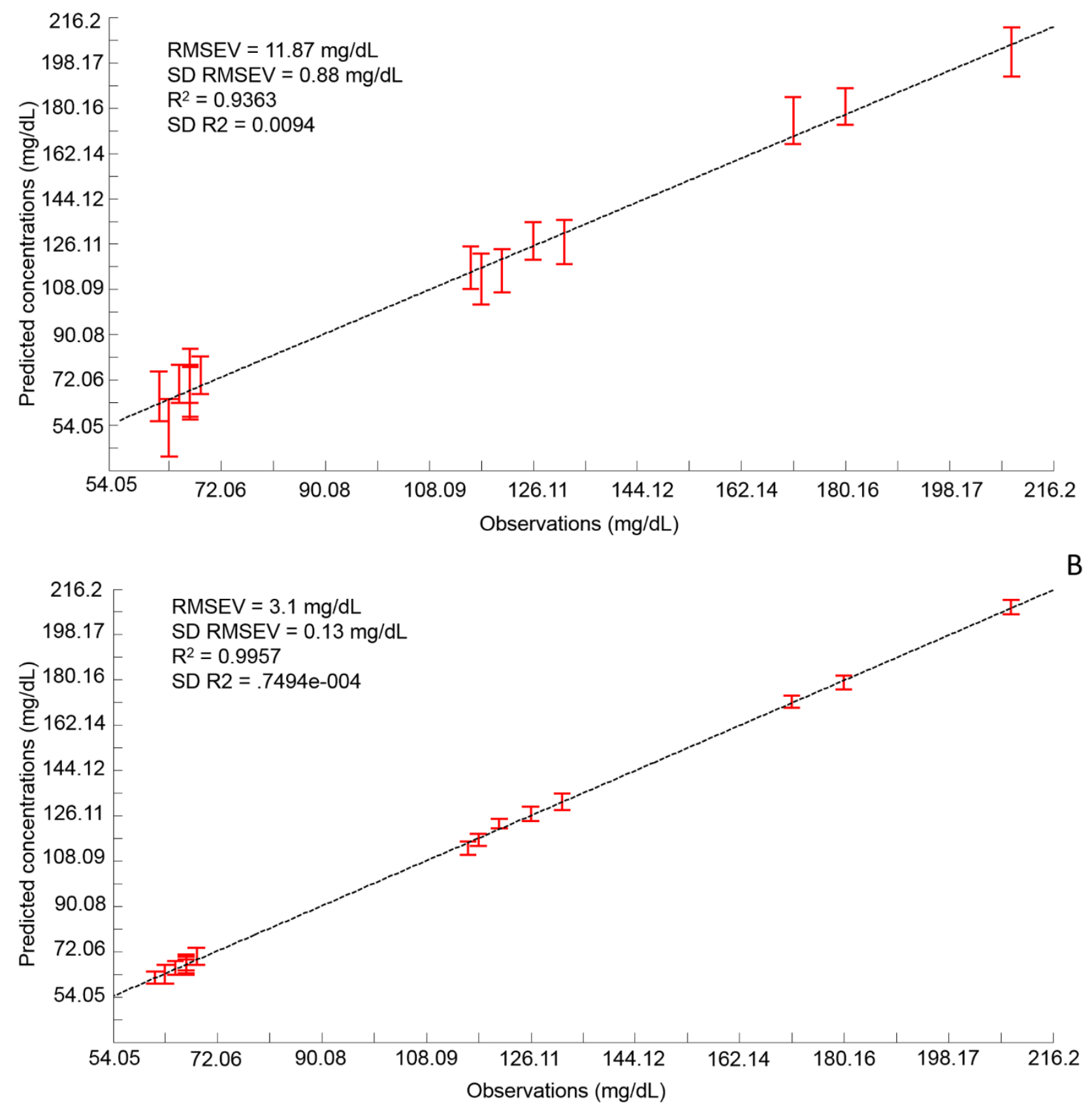

Figure 9: A: Predictive model built from the PLS analysis of patient unprocessed samples; B: Predictive model built from the PLS analysis of patient 10kda filtered samples. For each concentrations the value displayed is an average of the concentration predicted with the corresponding standard deviation calculated from the 20 iterations of the cross validation. Mean RMSEV and $R 2$ values are given on the plot both also with their respective standard deviation. Min-Max normalized at 1780 $\mathrm{cm}-1$. 

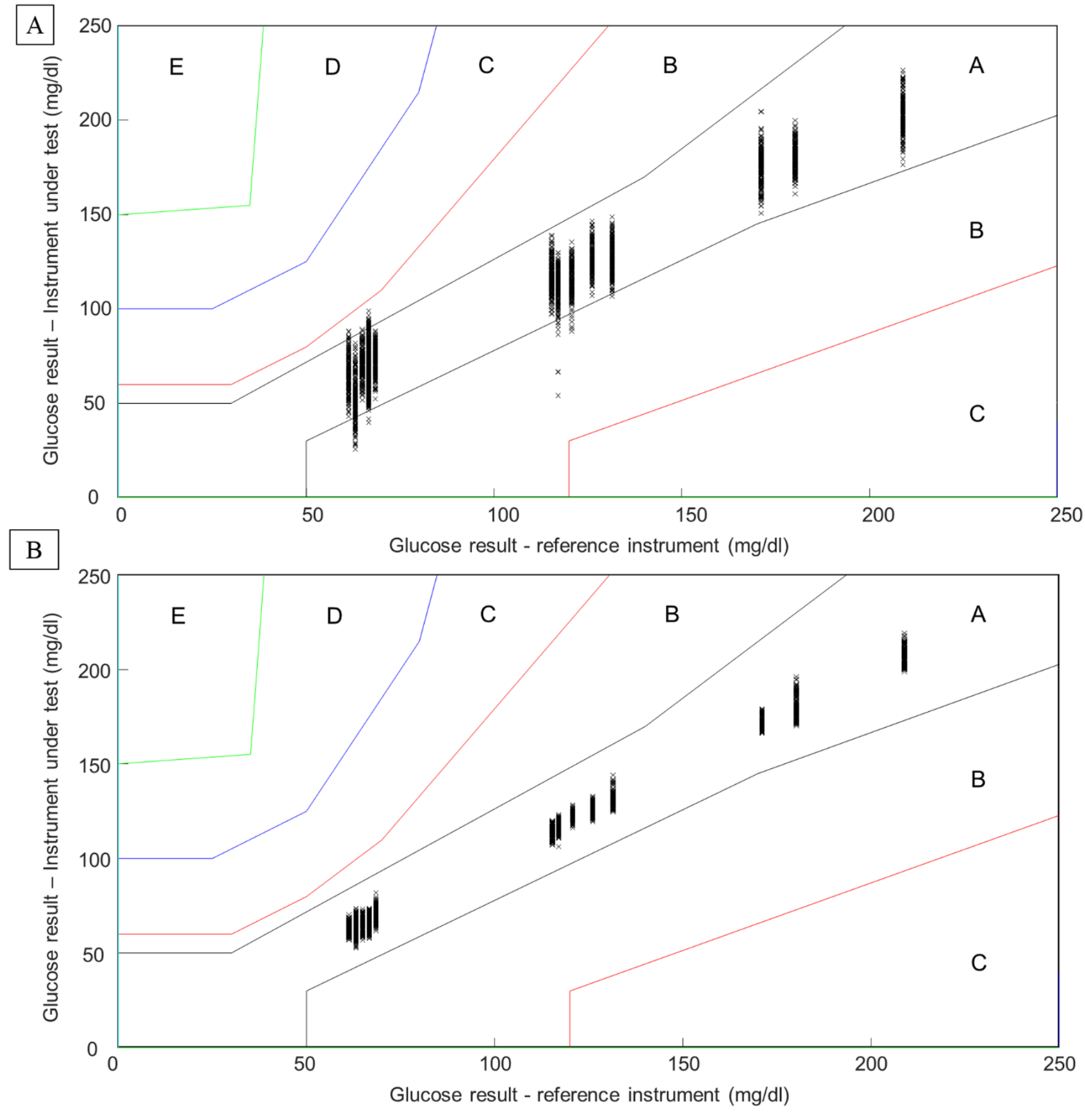

Figure 10: Parkes error grid for patient serum samples. A: Predicted values obtained from the PLSR analysis performed on the whole human serum from patients. B: Predicted values obtained from filtered human serum. Labels $A-E$ define the risks zone restricted to the range $0-250 \mathrm{mg} / \mathrm{dL}$ 


\section{Ultra-filtration of human serum for improved quantitative analysis of low molecular weight biomarkers using ATR-IR spectroscopy}

Franck Bonnier ${ }^{*}, 1$, Hélène Blasco ${ }^{2,3}$, Clément Wasselet $^{1}$, Guillaume Brachet $^{4}$, Renaud Respaud $^{5}$, Luis Felipe CS. Carvalho ${ }^{6}$, Dominique Bertrand ${ }^{7}$, Matthew J. Baker ${ }^{8}$, Hugh J. Byrne $^{9}$, Igor Chourpa ${ }^{1}$

${ }^{1}$ Université François-Rabelais de Tours, Faculté de Pharmacie, EA 6295 Nanomédicaments et Nanosondes, 31 avenue Monge, 37200 Tours, France.

${ }^{2}$ CHRU de Tours, Laboratoire de Biochimie et de Biologie Moléculaire, Tours, France.

${ }^{3}$ INSERM, UMR U930 "Imagerie et Cerveau", Université François Rabelais, Tours, France.

${ }^{4}$ Université François Rabelais de Tours, UMR CNRS 7292 Génétique, Immunothérapie, Chimie et Cancer, Faculté de Médecine, 10 Bd Tonnellé, 37032 Tours Cedex

${ }^{5}$ Université François-Rabelais de Tours, UMR 1100, CHRU de Tours, Service de Pharmacie, F-37032 Tours, France

${ }^{6}$ Universidade do Vale do Paraiba, Laboratory of Biomedical Vibrational Spectroscopy, Sao José dos Campos, Brazil

${ }^{7}$ Data Frame, Nantes, France

${ }^{8}$ WestCHEM, Technology and Innovation Centre, Department of Pure and Applied Chemistry, University of Strathclyde, 295 Cathedral Street, Glasgow G1 1XL, UK

${ }^{9}$ FOCAS Research Institute, Dublin Institute of Technology (DIT), Camden Row, Dublin 8, Ireland

\section{Supplementary information}

S.1 Data pre-processing: Baseline correction and vector normalisation

Figure S.1 illustrates examples of ATR-IR spectra baseline correction and vector normalisation.

The data collected from mixed pool human serum spiked with increasing concentrations of glucose exhibit similar backgrounds. The ATR minimises strong baseline distortion related to Mie scattering which leads to simplified data pre-processing requirements (Figure S.1 A). For instance, although the baseline correction is based on a rubber band algorithm, only 2 nodes at 1800 and $900 \mathrm{~cm}^{-1}$ are required in order to compensate for a slight offset that can be observed in the data ( Figure S.1 B). Finally, a standard vector normalisation is applied to scale up of the spectra for direct comparison and analysis by means of PLSR. 


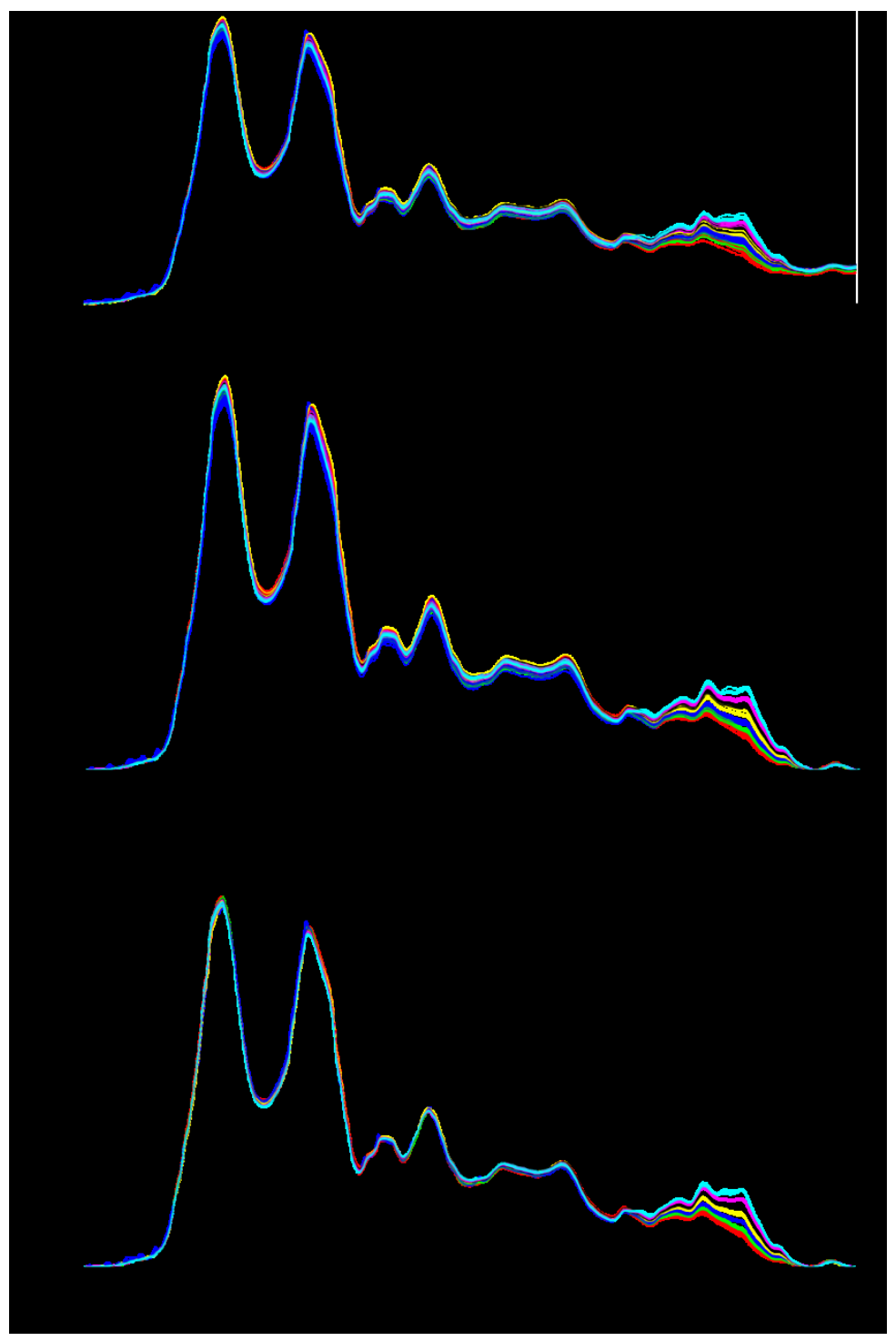

Figure S.1: Example of data processing. A: Raw infrared spectra collected from human serum spiked with different glucose concentrations. B: Same data set after baseline correction using the rubber band algorithm. C: Same data after vector normalisation. Spectra are colour coded as follows; Red: Human serum stock solution, Green: serum spiked with $20 \mathrm{mg} . \mathrm{dL}^{-1}$, Blue: serum spiked with $60 \mathrm{mg} . \mathrm{dL}^{-1}$, Yellow: serum spiked with $100 \mathrm{mg} \cdot \mathrm{dL}^{-1}$, Black: serum spiked with $140 \mathrm{mg} \cdot \mathrm{dL}^{-1}$, Magenta: serum spiked with $180 \mathrm{mg} \cdot \mathrm{dL}^{-1}$ and Cyan: serum spiked with 220 mg.dL ${ }^{-1}$. 


\section{S.2 Pure Glucose}

S.2.1. Minimum and maximum concentrations

In such studies, it is also interesting to address the question of the limit of detection for a given molecule, for instance glucose. While the PLSR model delivers information related to quantitative precision, working with air dried samples can lead to questions of the maximum and minimum of glucose concentrations which can be analysed. As documented previously, the behaviour of the spectral changes is not similar for low and high concentrations and thus the limitations need to be considered separately ${ }^{24}$. Figures S.2 A and B display correlations of the amount of glucose deposited on the crystal and the measured absorbance in the Area Under the Curve (AUC) for the glucose bands in the region 1180-955 $\mathrm{cm}^{-1}$. The Table in Figure S.3 gives an overview of the maximum and minimum concentrations of glucose measurable with ATRIR, depending on the drop size use for the recording. It is notable that, with a limit of detection at $0.015 \mu \mathrm{g}$, even the use of $2 \mu \mathrm{L}$ drops is limited to a minimum concentration of $0.75 \mathrm{mg} / \mathrm{dL}$. 

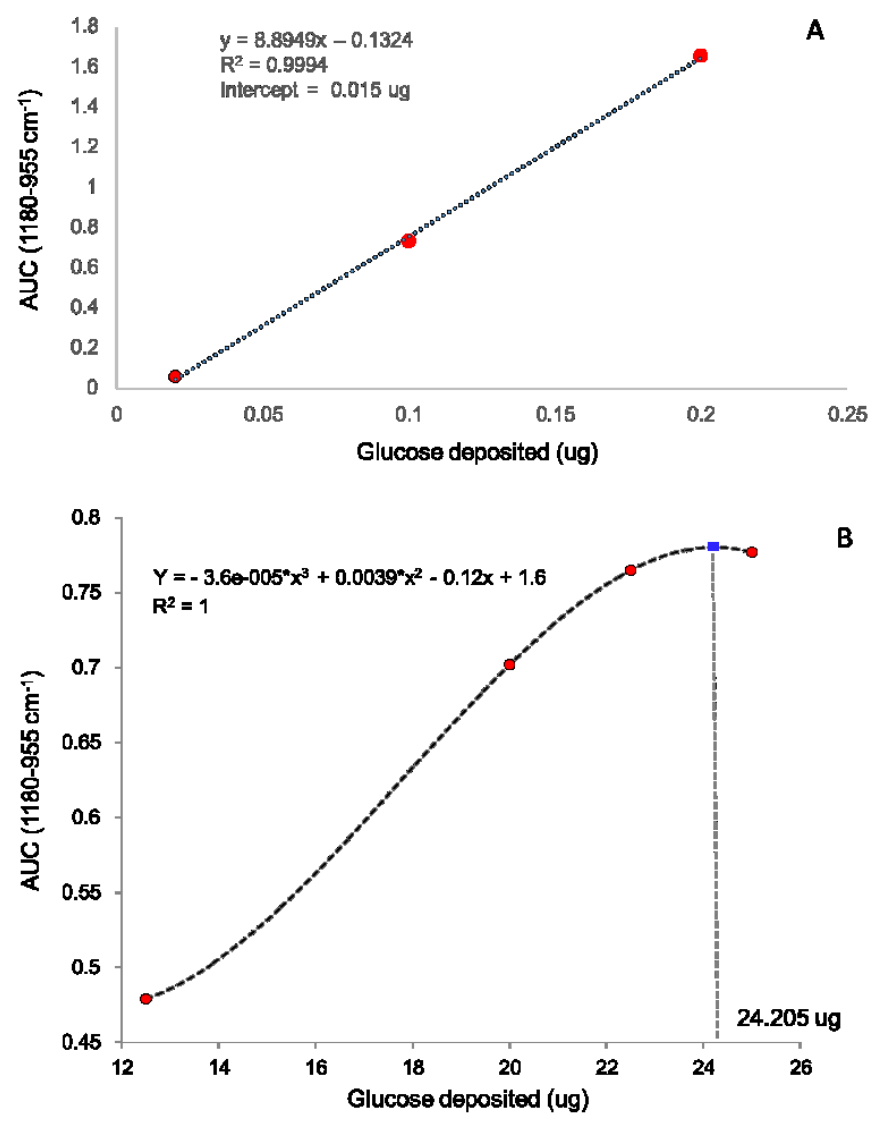

Figure S.2: Estimation of the limit of detection for ATR-IR analysis of glucose solutions. A: linear fitting for amount of glucose below $0.2 \mu \mathrm{g}$. B: Polynomial fitting for amount of glucose deposited above $12.5 \mu \mathrm{g}$.

\begin{tabular}{|c|c|c|}
\hline$V(\mu L)$ & $C_{\min }(\mathrm{mg} / \mathrm{dL})$ & $C_{\max }(\mathrm{mg} / \mathrm{dL})$ \\
\hline 0,1 & 15 & 24205 \\
\hline 0,2 & 7,5 & 12102 \\
\hline 0,5 & 3 & 4841 \\
\hline 1 & 1,5 & 2420 \\
\hline 2 & 0,75 & 1210 \\
\hline
\end{tabular}

Figure S.3: Summary minimum and maximum concentrations hat can be analysed depending on the drop size deposited on the ATR crystal. Concentrations expressed in $\mathrm{mg} / \mathrm{dL}$ for comparison with clinical results. 


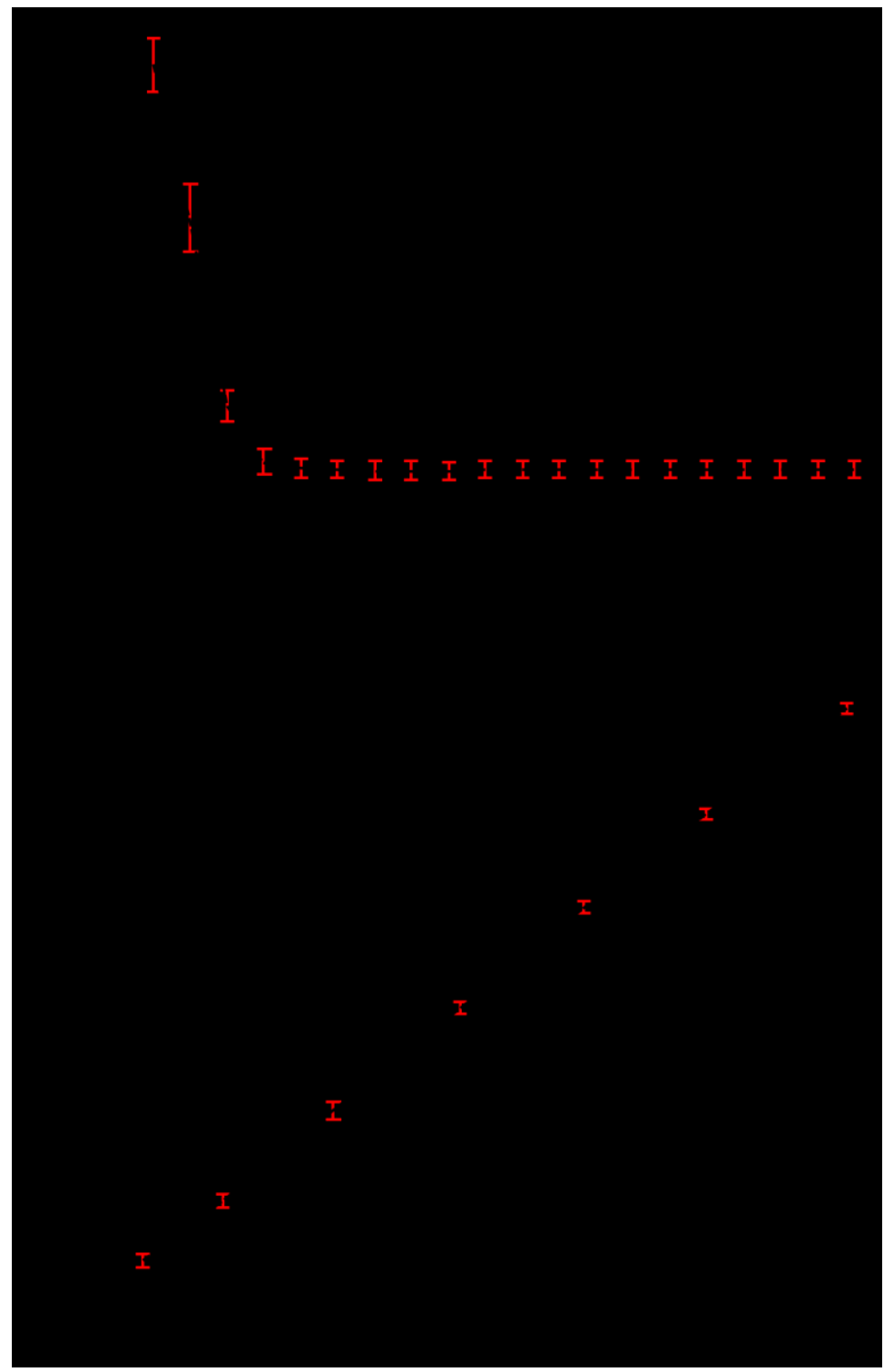

Figure S.4: PLSR model constructed from IR spectra collected from aqueous glucose solutions. Similar to patient samples, $2 \mu \mathrm{L}$ drops air dried before recording have been analysed. A: Evolution of the root mean square error on the validation set (RMSEV) according to the number of dimensions selected in the PLSR model. Values are averages calculated from the 20 iterations of the cross validation associated with the corresponding error bar illustrating the standard deviation. B: Predictive model built from the PLSR analysis. For each concentration, the value displayed is an average of the concentration predicted with the corresponding standard deviation calculated from the 20 iterations of the cross validation. Mean RMSEV and $\mathrm{R}^{2}$ values are given on the plot both also with their respective standard deviation. 